In Vitro Angiogenesis Inhibition with Selective Compounds Targeting the Key Glycolytic Enzyme PFKFB3

Anahita Abdali, Denisa Baci, Isabella Damiani, Federica Belloni, Carlo De Dominicis, Maria Luisa Gelmi, Alberto Corsini, Stefano Bellosta

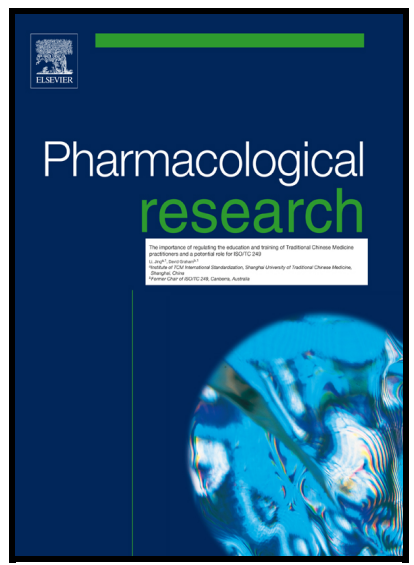

PII: $\quad$ S1043-6618(21)00176-6

DOI: $\quad$ https://doi.org/10.1016/j.phrs.2021.105592

Reference: YPHRS105592

To appear in: Pharmacological Research

Received date: 30 July 2019

Revised date: 27 March 2021

Accepted date: 28 March 2021

Please cite this article as: Anahita Abdali, Denisa Baci, Isabella Damiani, Federica Belloni, Carlo De Dominicis, Maria Luisa Gelmi, Alberto Corsini and Stefano Bellosta, In Vitro Angiogenesis Inhibition with Selective Compounds Targeting the Key Glycolytic Enzyme PFKFB3,Pharmacological Research, (2019) doi:https://doi.org/10.1016/j.phrs.2021.105592

This is a PDF file of an article that has undergone enhancements after acceptance, such as the addition of a cover page and metadata, and formatting for readability, but it is not yet the definitive version of record. This version will undergo additional copyediting, typesetting and review before it is published in its final form, but we are providing this version to give early visibility of the article. Please note that, during the production process, errors may be discovered which could affect the content, and all legal disclaimers that apply to the journal pertain.

(C) 2019 Published by Elsevier. 


\section{In Vitro Angiogenesis Inhibition with Selective Compounds Targeting the Key Glycolytic Enzyme PFKFB3}

Anahita Abdali ${ }^{1}$, Denisa Baci ${ }^{2}$, Isabella Damiani ${ }^{1}$, Federica Belloni ${ }^{1}$, Carlo De Dominicis ${ }^{3}$, Maria Luisa Gelmi ${ }^{4}$, Alberto Corsini ${ }^{1,5}$, Stefano Bellosta ${ }^{1}$

${ }^{1}$ Dept. of Pharmacological and Biomolecular Sciences, Università degli Studi di Milano, Milan, Italy

${ }^{2}$ Department of Biotechnology and Life Sciences, University of Insubria, Varese, Italy

${ }^{3}$ Institute of Medical Sciences, Aberdeen, University of Aberdeen, United Kingdom

${ }^{4}$ Dept. of Pharmaceutical Sciences, Università degli Studi di Milano, Milan, Italy

${ }^{5}$ IRCCS MultiMedica, Milan, Italy

\section{Corresponding author: Stefano Bellosta}

Dept. of Pharmacological and Biomolecular Sciences,

Università degli Studi di Milano

Via G. Balzaretti 9, 20133, Milan, Italy

E-mail address: stefano.bellosta@unimi.it,

\section{Abstract}

Abnormal glycolytic metabolism contributes to angiogenic sprouting involved in atherogenesis. We investigated the potential anti-angiogenic properties of specific 6phosphofructo-2-kinase/fructose-2,6-bisphosphatase-3 (PFKFB3) inhibitors in endothelial cells (ECs).

ECs were treated with PFKFB3 inhibitors (named PA-1 and PA-2) and their effects on metabolic and functional characteristics of ECs were investigated. The anti-glycolytic compound 3-(pyridinyl)-1-(4-pyridinyl)-2-propen-1-one (3PO) was used as reference compound.

PFKFB3 expression and activity $\left(\mathrm{IC}_{50}\right.$ about 3-21 nM) was inhibited upon treatment with both compounds. Glucose uptake and lactate export were measured using commercial assays and showed a partial reduction up to $40 \%$. PFKFB3 inhibition increased intracellular lactate accumulation, and reduced expression of monocarboxylate transporters-1 (MCT1) and MCT4. Furthermore, endothelial cell migration and proliferation assays demonstrated significant reduction upon treatment with both compounds. Matrix- metalloproteinase (MMP) activity, measured by gelatin zymography, and expression was significantly reduced (up to 25\%). In addition, PA compounds downregulated the expression of VCAM-1, VE-cadherin, VEGFa, VEGFR2, TGF- $\beta$, and IL-1 $\beta$, in inflamed ECs. Finally, PA-1 and PA-2 treatment 
impaired the formation of angiogenic sprouts measured by both morphogenesis and spheroid-based angiogenesis assays.

Our data demonstrate that the anti-glycolytic PA compounds may affect several steps involved in angiogenesis. Targeting the key glycolytic enzyme PFKFB3 might represent an attractive therapeutic strategy to improve the efficacy of cancer treatments, or to be applied in other pathologies where angiogenesis is a detrimental factor.

\section{Graphical abstract}

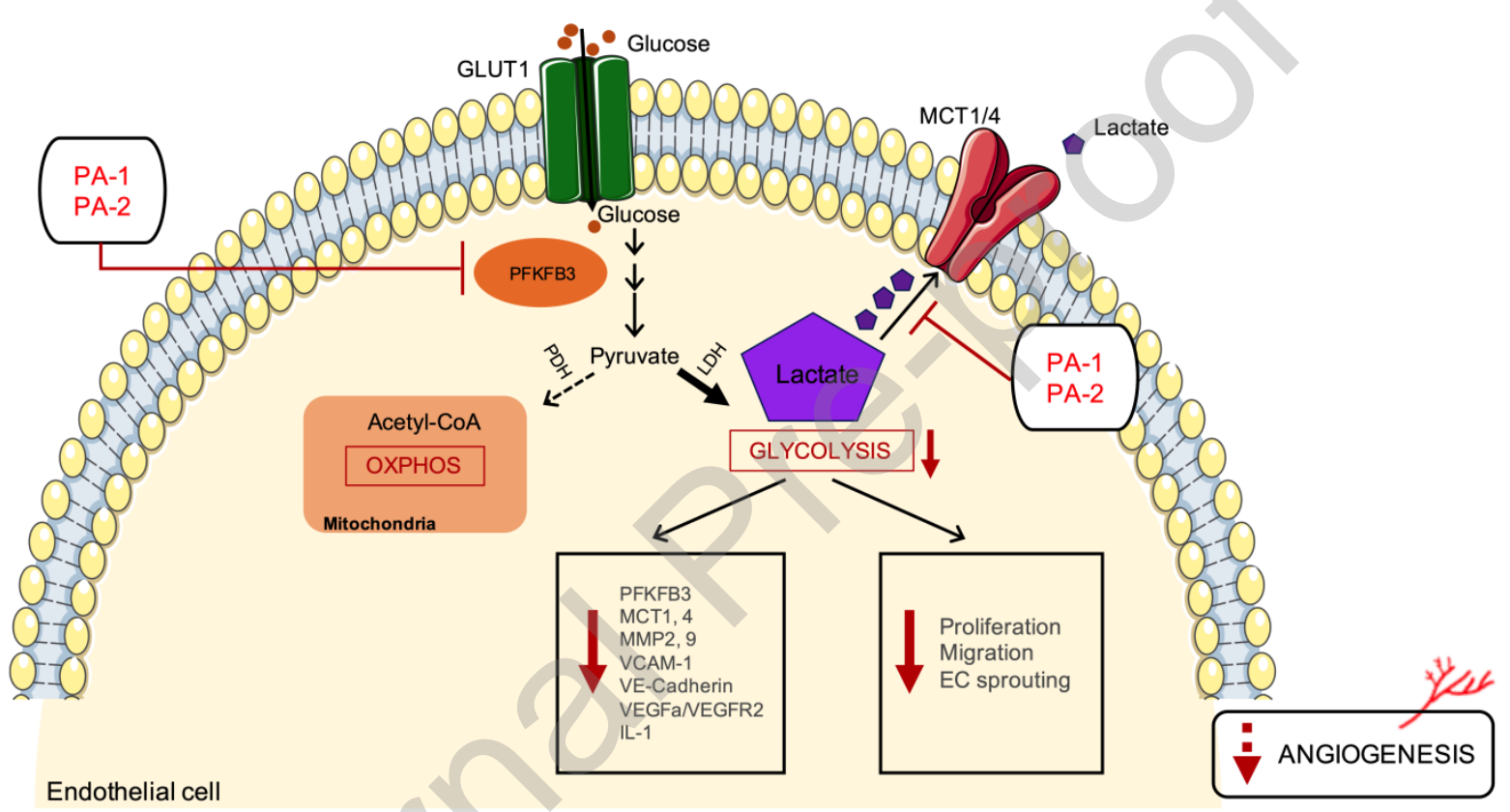

Abbreviations: 2-DG - 2-deoxyglucose; EC - endothelial cell; EHEC - EA.hy926 endothelial cell; HUVEC - human umbilical vein endothelial cell; MCT -monocarboxylate transporter; MitoC - mitomycin C; MMP - matrix metalloproteinase; NAD - nicotinamide adenine dinucleotide; OXPHOS - oxidative phosphorylation; PPP - pentose phosphate pathway; PA - phenoxindazole analogue; PFK-1 - 6-phospho-fructokinase-1; PFKFB3 - 6phosphofructo-2-kinase/fructose-2,6-bisphosphatase-3; 3PO - 3-(pyridinyl)-1-(4-pyridinyl)-2propen-1-one; TGF- $\beta$ - transforming growth factor beta; TNF- $\alpha$ - tumor necrosis factor alpha; VE-cadherin - vascular endothelial cadherin; VEGF - vascular endothelial growth factor; VEGFR2 - vascular endothelial growth factor receptor 2; VCAM - vascular cell adhesion molecule 
Keywords: PFKFB3 - Angiogenesis - Glycolysis - Monocarboxylate transporter (MCT) Matrix metalloproteinase (MMP) - Anti-angiogenic therapy

\section{Chemical compounds studied in this article.}

3PO (PubChem CID: 5720233); PA-1 (PubChem CID: 118735790); and PA-2 (PubChem CID: 118735791).

What is already known: Glycolytic modulation by inhibition of the enzyme PFKFB3 has been shown to reduce angiogenesis.

What this study adds: Insight into the inhibitory effects of novel and specific PFKFB3 inhibitors on glycolysis, migration, and angiogenesis.

Clinical significance: Significant inhibition of angiogenesis with selective PFKFB3 inhibitors could be a treatment of uncontrolled angiogenesis, and vasculogenesis.

\section{Introduction}

Angiogenesis is a highly dynamic and coordinated process consisting of the growth of new blood vessels from existing vasculature and is triggered by pro-angiogenic factors, such as vascular endothelial growth factor (VEGF), and an increased metabolism of endothelial cells (ECs). This is required for the supply of oxygen and nutrients, removal of waste, and immune surveillance. A balance between angiogenic and angiostatic factors regulates the growth and regression of new sprouts [1]. In response to injury/oxygen deprivation or pathological conditions, such as inflammation and tumor growth, ECs rapidly switch from a quiescent to a highly migratory and proliferative state $[2,3]$ as a result of release of VEGF and matrix metalloproteinases (MMPs; in particular MMP-2 and MMP-9) [2,4]. Current therapies targeting VEGF and its receptors are effective and safe, as demonstrated by the clinical use of bevacizumab as first line treatment in many tumors $[5,6]$. However, some limitations are 
still present due to the development of resistance and/or toxicity $[7,8]$. Therefore, alternative anti-angiogenic strategies are needed. New anti-angiogenic approaches focused on the inhibition of endothelial metabolism and glycolysis $[3,9]$ are emerging as appealing therapeutic strategies to treat pathological angiogenesis.

ECs have high glycolytic activity that is comparable to that of tumor cells. Out of this process, $85 \%$ of the total cellular ATP content is generated [10]. The conversion of fructose-6phosphate to fructose-1,6-bisphosphate is one of three rate-limiting steps regulated by 6phospho-fructokinase-1 (PFK-1). The bifunctional enzyme 6-phosphofructo-2kinase/fructose-2,6-bisphosphatase-3 (PFKFB3) is a direct and powerful activator of PFK-1 [10]. Of all PFKFB isoenzymes, PFKFB3 is most abundantly expressed in ECs [11]. Recent studies have shown the efficacy of partially reducing glycolysis, via PFKFB3 inhibition, as a way to impair vessel sprouting and reduce tumor growth [10-13]. Specifically, PFKFB3 was shown to be important for EC migration, proliferation and to be a powerful angiogenic regulator [10,13]. Given its key role as a glycolytic activator, pharmacological inhibition of PFKFB3 represents a promising anti-angiogenic strategy for targeting pathological angiogenesis, overcoming resistance and insufficient efficacy of classic anti-angiogenic therapies [13]. Although the anti-glycolytic compound 3-(pyridinyl)-1-(4-pyridinyl)-2-propen-1one (3PO) has shown promising results against angiogenesis, at high concentrations it compromises vascular barrier integrity [14] and has immunosuppressive functions [15]. In fact, 3PO facilitates cancer cell intravasation and dissemination thus leading to metastasis [14]. Furthermore, pharmacological activities of $3 \mathrm{PO}$ cannot be uniquely attributed to the inhibition of PFKFB3 and there are no evidences of binding to the PFKFB3 kinase domain $[16,17]$. Therefore, the development of selective inhibitors of PFKFB3, with low general toxicity, is needed.

Herein we tested the anti-angiogenic potential of two phenoxindazole analogues (PA-1 and PA-2) self-synthesized based on a previous paper by Boyd [16]. These compounds have a greater potency in inhibiting PFKFB3, as compared to the most widely studied agents, namely 3PO $[12,13,16,18]$ and PFK15 [18]. 
Results presented here demonstrate the ability of these more potent and selective inhibitors of PFKFB3 to block pathological angiogenesis in basal and inflamed conditions. Furthermore, we also investigated the possible mechanism(s) of action of these compounds in angiogenesis.

\section{Results}

\subsection{Novel PFKFB3 inhibitors suppress PFKFB3 and affect lactate metabolism}

The impact of pharmacological inhibition of the kinase activity of PFKFB3 on glycolysis levels were assessed in both an EC line (EHEC) and in primary ECs (HUVEC), widely used for cell based assays in the field of in vitro angiogenesis research [19]. In our study, key findings were confirmed by using 3PO as a reference compound. In the first series of experiments, we performed concentration-response studies to assess the most active nontoxic concentration of all compounds able to induce functional changes in EC behavior such as wound healing (Fig. S1a and S1C). In the following experiments, the PA compounds were tested at a nontoxic concentration of $20 \mu \mathrm{M}$ with a viability value of minimum 90 percent. The effectiveness of PA compounds (Fig. S1b) in suppressing PFKFB3 gene expression and protein level were then evaluated. Treatment with the PA compounds resulted in a significant reduction in PFKFB3 mRNA levels in EHECs (Fig. 1a) that was confirmed by immunoblot analyses of PFKFB3 protein levels (Fig. 1b). In contrast to these results, 3PO did not show any inhibitory effect on PFKFB3 transcripts although we observed a slight decrease at a protein level (Fig. 1a and 1b). These results were confirmed in human umbilical vein endothelial cells (HUVEC: Fig. 1c and 1d).

An elevated lactate concentration represents a good indicator of the metabolic adaptation of pathological angiogenesis and is actually correlated to clinical outcome in a variety of human cancers [20,21]. PFKFB3 regulates high glycolytic activity in ECs. In order to assess the metabolic effects of these PFKFB3 inhibitors on glycolysis we examined glucose uptake and lactate transport after PA treatment. Following reduced PFKFB3 expression, PA-1 and PA-2 
treatment markedly reduced the export of lactate (Fig. 1e), without having an impact on the intracellular levels of NAD+ and NAD+/NADH ratio (Fig. S1d). PA-2 was less effective, and a similar effect was observed with 3PO (Fig. 1e). Interestingly, we detected also a marked accumulation of lactate in EHECs upon treatment with both PA compounds, while the wellestablished PFKFB3 inhibitor 3PO was less effective (Fig. 1f). PFKFB3 inhibition by PA treatment also significantly decreased glucose uptake (Fig. 1g) in a dose-dependent manner. Collectively, these results suggest a potent regulatory effect of these newly synthesized compounds on glycolysis and lactate transport capacity in ECs.

\subsection{PFKFB3 inhibition regulates lactate transport through modulation of MCT1 and MCT4}

The main controllers of lactate fluxes in ECs are the monocarboxylate transporters (MCT) 1 and MCT4. MCT1 is known to be mainly a lactate importer to fuel the Krebs cycle, but can also act as an exporter, whereas MCT4 is known as a lactate exporter [22,23]. Therefore, we analyzed whether the observed effects on lactate levels are due to an alteration of the expression of MCT1 and/or MCT4. Expression analyses suggest that treatment with PA compounds significantly affected the expression of both MCT1 (Fig. 2a and 2c) and MCT4 (Fig. 2b and 2d) in both EC lines. Analysis of MCT1 and MCT4 protein level was less conclusive since treatment with PA compounds did not affect MCT1 levels (Fig. 2e and 2f), while we observed a downregulation of MCT4 protein level (Fig. $2 \mathrm{~g}$ ). These results collectively, suggest that PA compounds not only inhibit glycolysis by directly inhibiting PFKFB3 expression, but also substantially potentiated this effect by indirectly regulating lactate levels by modulating the expression of MCT1 and MCT4. 


\subsection{PFKFB3 inhibitors interfere with key steps of inflammatory angiogenesis:} proliferation, migration and protease activity

Endothelial cell proliferation and migration are crucial steps in inflammation-induced neovascularization. Previous studies already showed that PFKFB3 inhibition or knockdown suppress EC migration and proliferation [11,13,24,25].

In line with these findings, we confirmed that treatment with the PA compounds is capable of inhibiting EC proliferation (assessed by counting cell numbers) to a similar level (Fig. 3a) without affecting cell viability (Fig. S1c). Furthermore, PFKFB3 suppression with PA compounds impaired EC motility, as it reduced EC migration in a modified Boyden chamber (Fig. 3b), and directional migration in the absence and presence of the mitomycin C (MitoC: an inhibitor of cell proliferation) (Fig. 3c).

Notably, ECs treated with PA compounds expressed lower levels of endothelial activation markers such as vascular cell adhesion molecule 1 (VCAM-1: Fig. 3d and 3e) and vascular endothelial cadherin (VE-cadherin) (Fig. $3 \mathrm{~g}$ and $3 \mathrm{~h}$ ). Western blotting confirmed that VEcadherin expression was significantly inhibited by PFKFB3 inhibition on a protein level, although we did not observe any effect on VCAM-1 protein (Fig. 3f and 3i, respectively). These experiments indicate that endothelial PFKFB3 inhibition represses adhesion molecules production at a transcriptional level, which may cause an impaired migration. 3PO behaved ambiguously by reducing VCAM-1 and VE-cadherin expression in EHECs (Fig. 3d and 3h) but inducing VCAM-1 in HUVECs (Fig. 3e).

Due to the important role that MMPs (MMP-2 and MMP-9) have during angiogenesis [26], we sought to investigate whether PA compounds are capable of regulating the expression and activity of MMPs in ECs. As assessed by quantitative RT-PCR, we observed decreased MMP-2 and MMP-9 mRNA levels upon treatment with PA compounds (Fig. 4a and 4b). Alongside with these results, gelatin zymography revealed that treatment of ECs with PA compounds significantly reduced MMP-2 and MMP-9 activity (Fig. 4c-e). 3PO had a similar effect on the gelatinolytic activity (Fig. 4). 


\subsection{Novel PFKFB3 inhibitors impair angiogenic sprouting in vitro}

Angiogenesis and inflammation are important hallmarks of several pathological situations $[27,28]$. Mimicking the scenario occurring in a pro-angiogenic environment, we exposed ECs to VEGF and FGF2 and examined whether PFKFB3 inhibition with PA compounds may affect the capacity of ECs to induce the formation of capillary-like structures on matrigel. We demonstrated that both PA-1 and PA-2 significantly impaired VEGF-induced endothelial sprouting, as shown by the decreased number and length of master segments together with a reduced number and surface area of formed meshes in HUVECs (Fig. 5a). Similar effects were observed in EHECs, although these cell lines appear to be more sensitive to the treatment with PA compounds (Fig. S2). With use of EC spheroids, the effect of these PFKFB3 inhibitors was studied on vessel sprouting. As a critical regulator of vessel outgrowth, PFKFB3 modulation by PA-1 and PA-2 treatment shortened the length of the neovessels after 24 hours of incubation (Fig. 5b), while the effect of 3PO was less pronounced.

\subsection{PFKFB3 inhibition suppresses angiogenic phenotype of endothelial cells}

The endothelial VEGFA/VEGFR2 axis is the major pathway that regulates angiogenesis [2931]. We therefore investigated whether the anti-angiogenic activity of PA compounds is regulated through this pathway. RT-PCR and western blot analysis revealed that PA-treated ECs expressed reduced mRNA and protein levels of VEGFR2 (Fig. 6a and 6d, respectively) and VEGFA (Fig. 6b and 6e).

The cytokine tumor necrosis factor (TNF)- $\alpha$ is a potent mediator of inflammation and angiogenesis [32,33], as it has been demonstrated to activate VEGFR2 and downstream signaling transduction molecules such as PI3K and AKT known to regulate EC motility and angiogenesis $[34,35]$. Therefore, next, we identified the potential effect that PFKFB3 inhibition might have on the expression of important genes related to glycolysis, migration and angiogenesis in TNF-stimulated ECs. Similarly, to previous observations, expression analyses demonstrated that PA compounds inhibited TNF- $\alpha$-mediated induction of PFKFB3, 
MCT1 and MCT4 in ECs (Fig. 6f). Moreover, VCAM-1 and VE-cadherin expression was markedly reduced (Fig. 6f). Transforming growth factor beta (TGF- $\beta$ ) have been shown to promote the expression of VEGF and protect ECs from apoptosis [36,37]. To this end, we analyzed the expression of TGF- $\beta$ and observed a significant reduction upon PA treatment in basal and inflammatory conditions (Fig. $6 \mathrm{c}$ and 6f, respectively). Together with this, interleukin (IL)-1 $\beta$ expression was significantly inhibited in TNF- $\alpha$ activated ECs (Fig. 6f).

Taken together, these results indicate that PFKFB3 inhibition with PA compounds impairs angiogenesis in basal and inflamed conditions by affecting the VEGF/VEGFR2 pathway and interfering with crucial steps such as cell migration and proliferation.

\section{Discussion}

Since angiogenesis is essential in the pathophysiology of various diseases, such as cancer [38] and atherosclerosis [39], several anti-angiogenic treatment strategies have emerged and been applied in the clinic [40]. Traditional VEGF-targeted therapy, such as the monoclonal antibody bevacizumab, often fails to render sustained responses with minimal increased survival rate in treatment of several cancers due to the development of drug resistance [4043]. This underlines the need for novel anti-angiogenic therapeutic approaches with fundamentally different mechanisms of action.

A new paradigm for treatment of pathological angiogenesis is by targeting EC metabolism with an anti-glycolytic therapy through inhibition of the key metabolic enzyme PFKFB3 [44]. Mono- and combination therapy of the PFKFB3 inhibitor 3PO with VEGFR tyrosine kinase inhibitor SU5416 significantly reduced pathological angiogenesis in models of ocular disease and inflammatory bowel disease by partly lowering glycolysis (up to $40 \%$ ) [13]. Through an indirect inhibition of glycolysis, the metabolism of activated ECs can be normalized to a quiescent state. This approach has shown no harmful consequences at a systemic level, as opposed to direct and maximal inhibition of glycolysis (e.g. with 2-deoxyglucose) which 
elicited cell death $[45,46]$. Hence, a moderate inhibition of glycolysis promises to be an appropriate therapeutic strategy for aberrant angiogenesis.

As observed by others [16], we also confirmed that 3PO does not bind to PFKFB3 and does not show any activity in a PFKFB3 kinase assay (IC50 > $100 \mu \mathrm{M})$ [47]. This, together with the immunosuppressive effects of 3PO [15], suggests unidentified off-target effects of 3PO. Therefore, we selected two highly specific and potent PFKFB3 inhibitors, with validated PFKFB3 kinase binding activity [16] and studied their anti-angiogenic potential in ECs. PA-1 $\left(\mathrm{IC}_{50}=4 \mathrm{nM}\right)$ and PA-2 $\left(\mathrm{IC}_{50}=3 \mathrm{nM}\right)$ show potent inhibitory activity in a PFKFB3 kinase assay [16]. The PA compounds are very effective in inhibiting PFKFB3 activity and their $\mathrm{IC}_{50}$ is much lower than other known PFKFB3 inhibitors, such as PFK158 which has an $\mathrm{IC}_{50}$ of $137 \mathrm{nM}[48]$.

ECs rely on glycolysis, both in resting and activated state, rather than oxidative phosphorylation. As compared to macrophages, fibroblasts, cardiomyocytes and other vascular cell types, ECs have a much higher level of glycolysis (85\%) to generate energy. PFKFB3, the most abundant isoenzyme in ECs, has a critical role in EC glycolysis. Knockdown of PFKFB3 in ECs results in a decrease of glycolysis up to 40\% [10].

We first reported that pharmacological blockade of PFKFB3 with PA analogues reduced PFKFB3 levels. Consequently, glucose uptake and glycolysis were reduced up to $40 \%$, while the accumulation of intracellular lactate was enhanced. ECs cells co-express the lactate transporters MCT1 (importer) and MCT4 (exporter) that can passively and bidirectionally transport several monocarboxylate metabolites, such as lactate, pyruvate, D- $\beta$ hydroxybutyrate and acetoacetate across the plasma membrane. Interestingly, it has been demonstrated that MCT4 predominates over MCT1 for lactate export in highly glycolytic cells $[23,49]$. Overexpression of MCTs has been associated with enhanced cell proliferation, migration, invasion and angiogenesis and therefore they have been considered as promising targets for cancer therapy [23]. We observed that glycolysis inhibition via PFKFB3 leads to the accumulation of intracellular lactate, without causing intracellular acidification (data not shown). Consistent with this, Noble et al. provided compelling evidence that the 
accumulation of intracellular lactate can further reduce the glycolytic rate via negative feedback inhibition of the glycolytic enzyme phosphofructokinase-1 (PFK-1) [50]. This enzyme is regulated by PFKFB3 and several metabolic products including ATP, $\mathrm{H}+$, and lactate $[51,52]$. Altogether, it is likely that the reduced glycolysis we observed in our study is also sustained by a reduced expression of MCT1 and/or MCT4 that leads to an accumulation of cytosolic lactate, as suggested also in other studies [53,54]. Further research is needed to understand how lactate fluxes are regulated and if lactate metabolism interplays with other essential vascular and inflammatory cells in pathological angiogenesis.

Endothelial cells show a preference for glycolytic energy production, that may prepare for rapid sprouting and migration. In line with previous reports showing that PFKFB3 drives EC proliferation and migration $[10,11]$, we demonstrated that PFKFB3 inhibition with selective inhibitors impaired angiogenic sprouting in ECs by reducing EC proliferation, migration, and gelatinolytic activity. Alongside with this, the expression of EC adhesion molecules was lowered. Hence, by rendering ECs to be less adhesive, PFKFB3 inhibition in these cells hampers the development of sprouts $[55,56]$.

Endothelial PFKFB3 also has an essential role in pathological conditions, as TNF- $\alpha$, VEGF stimulation, and hypoxia exposure highly induce PFKFB3 expression $[10,51,57]$. Moreover, cytokines and growth factors are able to stimulate classical angiogenic signaling pathways, such as the VEGF/VEGFR2 pathway $[29,58]$. To this end, we have studied whether PFKFB3 inhibition with PA compounds could interfere with the VEGF/VEGFR2 signaling pathway in basal and inflammatory conditions. To mimic the vascular microenvironment in a proinflammatory setting, we activated ECs with TNF- $\alpha$. Our findings imply that PA treatment impairs essential drivers of pathological angiogenesis including proliferation, migration, and VEGFA/VEGFR2 signaling.

Whether PFKFB3 inhibition could induce a switch in ECs to aerobic metabolism, i.e. oxidative phosphorylation (OXPHOS), has not been studied yet. However, studies do indicate a shift in tumor cells from glycolysis toward the NADPH-producing pentose phosphate pathway (PPP) upon S-glutathionylation and demethylation of PFKFB3 that is 
induced by high levels of reactive oxygen species $[59,60]$. Also, 3PO was shown to induce PPP flux [13] which promotes the angiogenic progression [61,62]. Nicotinamide adenine dinucleotide $(\mathrm{NAD}+) /$ reduced $\mathrm{NAD}+(\mathrm{NADH})$ redox couple are important regulators of cellular metabolism including glycolysis and mitochondrial OXPHOS. Limiting levels of NAD+ together with DNA damage can drop glycolysis low enough to cause cell death, even in free access of glucose [63-65]. Remarkably, loss of NAD+ homeostasis has been linked to pathological conditions such as cardiovascular diseases, metabolic syndrome, and cancer [66-68]. However, a recent study demonstrated that elevating NADH levels does not affect angiogenic sprouting nor does it induce vessel formation in PFKFB3-silenced ECs [10]. Our analysis of intracellular NAD+ levels indicated that endothelial PFKFB3 inhibition by PA treatment maintained the $\mathrm{NAD+} / \mathrm{NADH}$ ratio, necessary for cell viability and to maintain physiological processes.

The development of anti-angiogenic agents is still an ongoing task. Standard PFKFB3 inhibitors, like 3PO and PFK15, have been the focus of several studies showing their therapeutic effects. However, these compounds are poorly specific or may lead to cell death. They are usually described to be active in the micromolar range, thereby necessitating maximum doses in preclinical models $[14,69]$. PA-1 and PA-2 were disclosed by AstraZeneca [16] and described to selectively inhibit lactate production by lung cancer cells. Big advantages of these PA compounds are their higher specificity and their ability to inhibit PFKFB3 activity and expression thus reducing glycolysis upstream of pyruvate formation. Also advantageous is their effect on endothelial phenotype through the inhibition of other glycolytic targets such as MCTs, together with adhesion and angiogenic molecules. For first time, we revealed that exposure of ECs to PFKFB3 inhibition regulates MMP-2 and MMP-9 activity (and expression). Overall, this suggests multiple and overlapping mechanisms of influence on angiogenesis by PA treatment.

PFKFB3 is highly expressed in atherosclerotic plaques [70]. One of the PA compounds has been used as PET tracer in animal models of atherosclerosis. The fluorinated form of PA-1 showed to be highly stable in the blood circulation and to target PFKFB3 in plaques located 
in mouse aorta and brachiocephalic arteries [71]. Altogether, the potential of PA-1 as a specific PFKFB3-targeted PET tracer has been successfully demonstrated for the detection of atherosclerotic plaques [71]. Future in vivo evaluation of these compounds will provide insights into their therapeutic activity and whether they qualify for clinical evaluation.

Inflammation and angiogenesis are important hallmarks of several diseases including atherosclerosis, pulmonary hypertension, and cancer [72-74]. Since the effect of PFKFB3 inhibition is not limited to endothelial cells, monocytes/macrophages, smooth muscle cells and $T$ cells are most likely also affected [75-77]. Attenuation of an increased glycolysis in many of these cell types is an effective strategy to slow their proliferation $[15,76,78]$, and in turn reduce tumor growth when applied to cancer models $[15,18]$ or affect mechanisms of vascular remodeling [76]. This study provides insight into the anti-angiogenic and antiinflammatory properties of PA compounds in endothelial cells, revealing a potential therapeutic application of these highly selective PFKFB3 inhibitors to treat aberrant angiogenesis and vasculogenesis.

\section{Materials and Methods}

\subsection{Cell Culture}

The human endothelial EA.hy926 cell (EHEC) line (ATCC CRL-2922) was cultured in high glucose Dulbecco's modified Eagle's medium (DMEM; EuroClone) supplemented with $10 \%$ Fetal Bovine Serum (FBS), $2 \mathrm{mM} \mathrm{L-Glutamine,} 100 \mathrm{U} / \mathrm{mL}$ penicillin, $100 \mu \mathrm{g} / \mathrm{mL}$ streptomycin, and 2\% HAT supplement (Sigma Aldrich) $[79,80]$. Primary human umbilical vein endothelial cells (HUVECs) were purchased from Lonza and grown in endothelial growth medium (EBM ${ }^{\mathrm{TM}}$; Lonza) supplemented with (EGM ${ }^{\mathrm{TM}}$ SingleQuots $^{\mathrm{TM}}$, Lonza), 10\% FBS, $2 \mathrm{mM} \mathrm{L-glutamine,} 100 \mathrm{U} / \mathrm{mL}$ penicillin and $100 \mathrm{mg} / \mathrm{mL}$ streptomycin and were used between passage 3-6. 


\subsection{Compounds}

The PFKFB3 inhibitor 3PO was purchased from Merck Millipore. The other two PFKFB3 inhibitors, called phenoxindazole analogue 1 (PA-1) and analogue 2 (PA-2) were described previously [16] and self-synthetized by us. The formal names of the compounds are listed below. All compounds were dissolved in dimethylsulphoxide (DMSO). EHECs were treated with $20 \mu \mathrm{M}$ 3PO, PA-1 and PA-2, while HUVECs were treated with $20 \mu \mathrm{M} 3 \mathrm{PO}, 5 \mu \mathrm{M}$ PA-1 and $20 \mu \mathrm{M}$ PA-2 for 24 hours. $20 \mu \mathrm{M}$ of PA-1 was cytotoxic in HUVECs.

$3 P O$ 3-(3-pyridinyl)-1-(4-pyridinyl)-2E-propen-1-one

PA-1 (2S)-N-(4-\{[3-cyano-1-(2-methylpropyl)-1H-indazol-5-yl]oxy\}phenyl) pyrrolidine-2-carboxamide

PA-2 (2S)-N-[4-(\{3-cyano-1-[(dimethyl-1,2-oxazol-4-yl)methyl]-1H-indazol-5yl\}oxy)phenyl] pyrrolidine-2-carboxamide

\subsection{Viability assay: LDH}

Cell viability was measured by using the CyQUANT LDH Cytotoxicity assay (Invitrogen). EHECs were seeded in 96-well plates at an equal density and treated at different concentrations of compounds for 24 hours. Following the manufacturer's instructions, absorbance of the cellular supernatant was measured at 490 and $680 \mathrm{~nm}$.

\subsection{Lactate measurements}

The levels of secreted lactate in EHECs were measured using the Glycolysis CellBased Assay Kit (Cayman Chemical), and the intracellular lactate concentration were measured with the L-Lactate Assay Kit (Cayman Chemical), following the manufacturer's protocol. Data were normalized to the protein concentration as quantified with the Pierce BCA Protein assay kit (Thermo Fisher Scientific). 


\subsection{NAD+ and NADH determination}

Intracellular NAD+ and NADH levels were measured using the NAD/NADH assay kit (Abcam; ab65348) according the manufacturer's protocol. Briefly, compound-treated endothelial cells were harvested by scraping. Cells were washed with cold PBS, and after centrifugation the pellet was used to extract total NAD/NADH. To detect NADH, samples were then decomposed by heating at $60^{\circ} \mathrm{C}$ for 30 minutes and quickly cooling samples on ice. Absorbance was measured at $450 \mathrm{nM}$ using a microplate reader to calculate the concentration of $\mathrm{NAD}+$ and $\mathrm{NADH}$, and subsequently the $\mathrm{NAD+} / \mathrm{NADH}$ ratio.

\subsection{Glucose uptake}

Glucose uptake was determined using the Glucose Uptake Cell-Based Assay Kit (Cayman Chemical) following the manufacturer's instructions. Briefly, EHECs were plated in complete medium in 96-well plates. Cells were then washed with PBS and treated with compounds for 24 hours in glucose-free medium (DMEM (GIBCO) supplemented with 10\% FBS, $1 \mathrm{mM}$ sodium pyruvate, $100 \mathrm{U} / \mathrm{mL}$ penicillin, and $100 \mu \mathrm{g} / \mathrm{mL}$ streptomycin; EuroClone), followed by 2-Deoxyglucose $(100 \mu \mathrm{g} / \mathrm{mL})$ incubation for 1 hour. Apigenin $(50 \mu \mathrm{M})$ and insulin (100nM) were used as positive controls. After treatment, supernatant was removed and 100 $\mu$ of cell-based assay buffer was added to each well. 2-Deoxyglucose taken up by cells was quantified with fluorescence filters at excitation and emission wavelengths of $485 \mathrm{~nm}$ and $535 \mathrm{~nm}$, respectively.

\subsection{Cell proliferation}

EHECs were seeded in $35 \mathrm{~mm}$ dishes in triplicate at an equal density. Next day, the medium was replaced with DMEM containing $0.4 \%$ FBS to starve and synchronize the cells. After 48 hours of starvation, ECs were treated with or without $20 \mu \mathrm{M} 3 \mathrm{PO}, \mathrm{PA}-1$ or PA-2 in culture medium for 24 hours. Cell numbers were counted using a counter analyzer (Beckman Coulter, Life Scientific), and compared to time-point zero. 


\subsection{In vitro directional migration (wound healing assay)}

EHECs were seeded in a 12-well plate and grown to confluence. To evaluate the responses in absence of proliferation, cells were pretreated with mitomycin $C(1 \mu \mathrm{g} / \mathrm{mL}$ : Sigma) for 24 hours. Monolayers were treated with or without the compounds in culture medium for 24 hours. Immediately after addition of the treatments, a scratch was made on the cell monolayer with a $200-\mu \mathrm{L}$ pipet tip through the middle of the well. After the wounding, images of the wounded area were captured of three to four random fields at $0 \mathrm{~h}$ and $24 \mathrm{~h}$ using an inverted microscope (Axiovert 200; Carl Zeiss, 20x objective lens) equipped with a digital camera. Quantification of the wound area was performed using ImageJ. All experiments were performed in triplicate and repeated two to three times.

\subsection{Chemotaxis}

EHEC chemotaxis was assayed using a modified microchemotaxis Boyden chamber. Polycarbonate filters (pore size: $8 \mu \mathrm{M}$; Transwell; Corning) were coated with $10 \mu \mathrm{g} / \mathrm{mL}$ fibronectin (Sigma). Cells were starved for 24 hours in DMEM containing $0.4 \%$ FBS and seeded at $10^{6}$ cells $/ \mathrm{mL}$ in the upper compartments together with the compounds. In the lower compartment, wells were filled with medium with the chemoattractant, serum. Endothelial cells were allowed to migrate toward $10 \% \mathrm{FCS}$ for $18 \mathrm{~h}$ at $37^{\circ} \mathrm{C}$ and $5 \% \mathrm{CO}_{2}$. Migrated cells were fixed and stained with the Diff-Quik staining set (Medion Diagnostics). Pictures were taken from at least three random fields for each experimental group with an inverted microscope (Axiovert 200; Carl Zeiss, 20x objective lens) equipped with a digital camera. Chemotaxis was quantified by counting the number of stained cells that migrated to the lower side of the filter. Stained cells without visible nucleus were excluded from the study.

\subsection{Gelatin gel zymography}

The effect of compounds on gelatinolytic activity was measured as previously described [81]. Briefly, EHECs were seeded in 48-well plates and treated with or without 
3PO, PA-1 and PA-2 in culture medium without serum for 24 hours. Then, conditioned medium was collected, and cell monolayers processed for evaluating cell protein content or mRNA extraction. Aliquots of conditioned medium $\left(30 \mu \mathrm{L}\right.$ per lane) were run at $4^{\circ} \mathrm{C}$ on $7.5 \%$ polyacrylamide gels containing $10 \%$ SDS and $1 \mathrm{mg} / \mathrm{mL}$ gelatin (Sigma-Aldrich) under nonreducing conditions and without boiling. After electrophoresis, the proteins were renatured by washing the SDS away in 2 washes with Triton X-100 (2.5\%) at room temperature. Hereafter, the gels were incubated overnight at $37^{\circ} \mathrm{C}$ in developing buffer $(50 \mathrm{mM}$ Tris- $\mathrm{HCl}$, $\mathrm{pH} 7.5$ containing $10 \mathrm{mM} \mathrm{CaCl}_{2}, 150 \mathrm{mM} \mathrm{NaCl}$, and $1 \mu \mathrm{M} \mathrm{ZnCl}_{2}$ ) to activate the gelatinases. Gels were subsequently stained with a solution of Coomassie brilliant blue $(0.1 \%$ Coomassie R250 in 25\% methanol, $7 \%$ acetic acid; Sigma-Aldrich) for 2 hours with gentle agitation, followed by destaining ( $25 \%$ methanol, $7 \%$ acetic acid) until areas of gelatinolytic activity appeared as transparent bands against a blue background. Finally, the gels were scanned using Chemidoc MP Imaging System (Biorad) and quantified by densitometric analyses using ImageJ software (National Institutes of Health). Values obtained were normalized to protein content, using the Pierce BCA Protein assay kit (Thermo Fisher Scientific), and expressed as a percentage of control.

\subsection{Western blotting}

Endothelial cells were treated with 3PO, PA-1 and PA-2 in culture medium for 24 hours. After an hour incubation, human TNF-a (10 ng/mL; Sigma, T0157) was added to induce inflammation. For the preparation of total cell lysates, cells were washed in cold PBS and lysed in ice-cold buffer composed of $50 \mathrm{mM}$ Tris- $\mathrm{HCl}(\mathrm{pH} 7.5), 150 \mathrm{mM} \mathrm{NaCl}, 0.5 \%$ Nonidet (NP-40), and protease inhibitors. Molecular mass marker (Sharpmass VI Prestained Protein Marker, Euroclone S.p.A) and proteins were separated through 4-12\% SDS-PAGE gel. Proteins were then transferred on to a nitrocellulose membrane and blocked with buffer containing $5 \%$ milk in $0.05 \%$ TBS-Tween. Membranes were probed with antibodies specific for PFKFB3 (1:1000, Abcam, ab96699), VE-cadherin (1:1000, Cell Signaling, 2158S), VEGFR2 (1:1000, Thermo Fisher Scientific, PA5-16487), MCT1 (Abcam ab85021, 1:1000), 
MCT4 (Proteintech 22787-1-AP, 1:1000), VCAM-1 (ab134047, 1:1000) and $\beta$-actin (1:1000, Sigma, A2228) overnight at $4{ }^{\circ} \mathrm{C}$. Next day, membranes were then incubated with goat antimouse 680RD and goat anti-rabbit $800 \mathrm{CW}$ IRDye secondary antibodies (1:10000, LI-COR Biosciences) for 1 hour at room temperature. Immunoreactive bands were detected by acquiring images with Odyssey ${ }^{\circledR}(\mathrm{LI}-\mathrm{COR}$ Biosciences). Densitometric readings were evaluated using the Image Studio Software.

\subsection{RNA isolation and real-time RT-PCR}

Endothelial cells were treated for 24 hours with or without $3 \mathrm{PO}, \mathrm{PA}-1$ or PA-2 in culture medium. To induce inflammation, after an hour incubation with the treatment the cells were stimulated with human TNF- $\alpha$ (10 ng/mL; Sigma, T0157). Total RNA was isolated using the Direct-zol ${ }^{\mathrm{TM}}$ RNA MiniPrep Pus kit (Zymo Research) and cDNA was generated by reverse transcription with the iScript gDNA Clear cDNA Synthesis Kit (Biorad), following the manufacturer's instructions. Real-time PCR was performed using iTaq $^{\text {TM }}$ Universal SYBR ${ }^{\circledR}$ Green supermix (Biorad) with gene-specific forward and reverse primers in an $A B I$ PRISM 7000 Sequence detection system (Applied Biosystems). Relative gene expression was converted using the $2^{-\triangle \Delta C T}$ method against the internal control large ribosomal phosphoprotein P0. The primer sequences are listed in Table 1. 


\begin{tabular}{|c|c|c|}
\hline Target gene & Forward primer $\left(\mathbf{5}^{\prime} \rightarrow \mathbf{3}^{\prime}\right)$ & Reverse primer $\left(\mathbf{5}^{\prime} \rightarrow \mathbf{3}^{\prime}\right)$ \\
\hline$R P L P 0$ & TCG ACA ATG GCA GCA TCT AC & ATC CGT CTC CAC AGA CAA GG \\
PFKFB3 & GAT GCC CTT CAG GAA AGC CT & GAA CAC TTT TGT GGG GAC GC \\
$M C T 1$ & CAC CGT ACA GCA ACT ATA CG & CAA TGG TCG CCT CTT GTA GA \\
$M C T 4$ & GAG TTT GGG ATC GGC TAC AG & CGG TTC ACG CAC ACA CTG \\
$M M P-2$ & TCT GTG TTG TCC AGA GGC AA & GCC TAA CAT CAT CAC TAG GCC A \\
$M M P-9$ & GTA CTC GAC CTG TAC CAG CG & AGA AGC CCC ACT TCT TGT CG \\
$I L-1 B$ & ATG CAC CTG TAC GAT CAC TG & ACA AGG ACA TGG AGA ACA CC \\
TGF- $\beta$ & CAG CAA CAA TTC CTG GCG ATA & AAG GCG AAA GCC CTC AAT TT \\
$V E G F A$ & ACG AAA GCG CAA GAA ATC CC & GGA GGC TCC AGG GCA TTA G \\
$V E G F R 2$ & ATG CAT CCT TGC AGG ACC AA & GGT TTC CTG TGA TCG TGG GT \\
$V C A M$ & GGA CCA CAT CTA CGC TGA CAA & GAC TGT GAT CGG CTT CCC AG \\
$V E C A D H E R I N$ & ACC AGG ACG CTT TCA CCA TT & GGC TCA TGT ATC GGA GGT CG \\
\hline
\end{tabular}

\section{Table 1. List of real-time PCR primer sequences}

\subsection{In vitro tube formation}

The effects of 3PO, PA-1 and PA-2 on endothelial cell ability to form capillary-like structures on basement membrane matrix was assessed in vitro, using the morphogenesis assay. EHECs and HUVECs were grown till $80 \%$ confluency in complete culture medium and serum starved overnight. Using pre-cooled tips and 96-well plates, $75 \mu \mathrm{L} /$ well of liquid matrigel (GFR Membrane Matrix; Corning \#356230) was distributed and allowed to solidify at $37^{\circ} \mathrm{C}$ for at least 30 minutes. Endothelial cells were cultured on the matrigel at a density of $1.5-2 \times 10^{5}$ in DMEM/EBM2 medium containing $100 \mathrm{ng} / \mathrm{mL}$ VEGF and $100 \mathrm{ng} / \mathrm{mL}$ FGF2 alone or with $3 \mathrm{PO}$ or PA-1 or PA-2 each. Pictures were captured after six hours for HUVECs, and 24 hours for EHECs using a Zeiss Microscope equipped with a Nikon camera (Axio Observer A1, Zeiss, Germany), and quantified using ImageJ software and the "Angiogenesis Analyzer" tool. 


\subsection{Spheroid-based sprouting angiogenesis}

Endothelial spheroids were prepared as described [82]. Briefly, HUVECs were suspended in growth medium containing $20 \%$ methocel (Sigma-Aldrich) and cultured as hanging drops (approximately 400 cells/spheroid) for 24 hours to form spheroids. Next, spheroids were resuspended in methocel containing 20\% FBS and transferred to collagen type I gels (Rat tail, Type I, Invitrogen) with a final concentration of $2.5 \mathrm{mg} / \mathrm{ml}$ and basal culture medium supplemented with VEGF-A and FGF (final concentration $25 \mathrm{ng} / \mathrm{ml}$; Peprotech). After 24 hours, spheroids were fixed with 4\% of paraformaldehyde.

Image analysis was done by quantifying the lengths of all sprouts of at least 8 spheroids per condition that were measured by using a Fiji software as previously described [82].

\subsection{Flow cytometry}

HUVECs were treated with the three compounds, 3PO, PA-1 and PA-2 in culture EBM-medium for 24 hours. Where after cells were detached, and cells were fixed, and permeabilized with CytoFix/Cytoperm kit (BD Biosciences) accordingly to manufacturer. Cells were washed and stained with PE-conjugated VEGFa (IC2931P, R\&D Systems) for 30 minutes at $4^{\circ} \mathrm{C}$. Fluorescence intensity was measured using a BD FACSCanto II flow cytometer. Experiments were performed on HUVECs activated with TNF- $\alpha$ (10ng/mL). Flow data were analyzed with FlowJo (Treestar Inc.). VEGFa-positive cells were quantified by the mean fluorescence intensity (MFI).

\subsection{Data and statistical analysis}

The presented data were derived from at least three independent experiments and are expressed as mean \pm SEM. Statistical significance was estimated with Student's t test for unpaired observations or ANOVA followed by the Bonferroni test when appropriate. 
Differences with values of $\mathrm{P}<0.05$ were considered statistically significant. Statistical analysis and graphical presentations were performed using GraphPad Prism 2016.

\section{Author contributions}

Designed the experiments: AA, SB. Performed the experiments: AA, DB, ID, FD. Analyzed the data: AA. Contributed reagents/tools: SB, AC, CDD, MLG. Wrote the paper: $A A, S B$.

\subsection{Conflicts of interest}

The authors declare no conflicts of interest.

\subsection{Acknowledgements}

This study was supported by funding from the European Union's HORIZON 2020 research and innovation programme under the Marie Skłodowska-Curie grant agreement number 675527.

\section{References}

[1] N. Ferrara, Pathways mediating VEGF-independent tumor angiogenesis, Cytokine Growth Factor Rev. 21 (2010) 21-26. doi:10.1016/J.CYTOGFR.2009.11.003.

[2] T.H. Adair, J.-P. Montani, Angiogenesis, Morgan \& Claypool Life Sciences, 2010. https://www.ncbi.nlm.nih.gov/books/NBK53238/.

[3] S. Vandekeere, M. Dewerchin, P. Carmeliet, Angiogenesis Revisited: An Overlooked Role of Endothelial Cell Metabolism in Vessel Sprouting, Microcirculation. 22 (2015) 509-517. doi:10.1111/micc.12229.

[4] V.W.M. Van Hinsbergh, P. Koolwijk, Endothelial sprouting and angiogenesis: Matrix metalloproteinases in the lead, Cardiovasc. Res. 78 (2008) 203-212. doi:10.1093/cvr/cvm102.

[5] E. Van Cutsem, F. Rivera, S. Berry, A. Kretzschmar, M. Michael, M. Dibartolomeo, M.A. Mazier, J.L. Canon, V. Georgoulias, M. Peeters, J. Bridgewater, D. Cunningham, Safety and efficacy of first-line bevacizumab with FOLFOX, XELOX, FOLFIRI and fluoropyrimidines in metastatic colorectal cancer: The BEAT study, Ann. Oncol. 20 (2009) 1842-1847. doi:10.1093/annonc/mdp233.

[6] D.W. Miles, V. Diéras, J. Cortés, A.A. Duenne, J. Yi, J. O’Shaughnessy, First-line 
bevacizumab in combination with chemotherapy for HER2-negative metastatic breast cancer: Pooled and subgroup analyses of data from 2447 patients, Ann. Oncol. 24 (2013) 2773-2780. doi:10.1093/annonc/mdt276.

[7] K. Kumar, S. Wigfield, H.E. Gee, C.M. Devlin, D. Singleton, J.-L. Li, F. Buffa, M. Huffman, A.L. Sinn, J. Silver, H. Turley, R. Leek, A.L. Harris, M. Ivan, Dichloroacetate reverses the hypoxic adaptation to bevacizumab and enhances its antitumor effects in mouse xenografts, J. Mol. Med. 91 (2013) 749-758. doi:10.1007/s00109-013-0996-2.

[8] G. Bergers, D. Hanahan, Modes of resistance to anti-angiogenic therapy, Nat. Rev. Cancer. 8 (2008) 592-603. doi:10.1038/nrc2442.

[9] G. Jiménez-Valerio, O. Casanovas, Angiogenesis and Metabolism: Entwined for Therapy Resistance., Trends in Cancer. 3 (2017) 10-18. doi:10.1016/j.trecan.2016.11.007.

[10] K. De Bock, M. Georgiadou, S. Schoors, A. Kuchnio, B.W. Wong, A.R. Cantelmo, A. Quaegebeur, B. Ghesquière, S. Cauwenberghs, G. Eelen, L.K. Phng, I. Betz, B. Tembuyser, K. Brepoels, J. Welti, I. Geudens, I. Segura, B. Cruys, F. Bifari, I. Decimo, R. Blanco, S. Wyns, J. Vangindertael, S. Rocha, R.T. Collins, S. Munck, D. Daelemans, H. Imamura, R. Devlieger, M. Rider, P.P. Van Veldhoven, F. Schuit, R. Bartrons, J. Hofkens, P. Fraisl, S. Telang, R.J. Deberardinis, L. Schoonjans, S. Vinckier, J. Chesney, H. Gerhardt, M. Dewerchin, P. Carmeliet, Role of PFKFB3driven glycolysis in vessel sprouting, Cell. 154 (2013) 651-663. doi:10.1016/j.cell.2013.06.037.

[11] Y. Xu, X. An, X. Guo, T.G. Habtetsion, Y. Wang, X. Xu, S. Kandala, Q. Li, H. Li, C. Zhang, R.B. Caldwell, D.J. Fulton, Y. Su, M.N. Hoda, G. Zhou, C. Wu, Y. Huo, Endothelial PFKFB3 plays a critical role in angiogenesis, Arterioscler. Thromb. Vasc. Biol. 34 (2014) 1231-1239. doi:10.1161/ATVBAHA.113.303041.

[12] L. Pisarsky, R. Bill, E. Fagiani, S. Dimeloe, R.W. Goosen, J. Hagmann, C. Hess, G. Christofori, Targeting Metabolic Symbiosis to Overcome Resistance to Anti-angiogenic Therapy, Cell Rep. 15 (2016) 1161-1174. doi:10.1016/j.celrep.2016.04.028.

[13] S. Schoors, K. De Bock, A.R. Cantelmo, M. Georgiadou, B. Ghesquière, S. Cauwenberghs, A. Kuchnio, B.W. Wong, A. Quaegebeur, J. Goveia, F. Bifari, X. Wang, R. Blanco, B. Tembuyser, I. Cornelissen, A. Bouché, S. Vinckier, S. DiazMoralli, H. Gerhardt, S. Telang, M. Cascante, J. Chesney, M. Dewerchin, P. Carmeliet, Partial and transient reduction of glycolysis by PFKFB3 blockade reduces pathological angiogenesis, Cell Metab. 19 (2014) 37-48. doi:10.1016/j.cmet.2013.11.008.

[14] L.-C. Conradi, A. Brajic, A.R. Cantelmo, A. Bouché, J. Kalucka, A. Pircher, U. Brüning, L.-A. Teuwen, S. Vinckier, B. Ghesquière, M. Dewerchin, P. Carmeliet, Tumor vessel disintegration by maximum tolerable PFKFB3 blockade, Angiogenesis. 20 (2017) 599613. doi:10.1007/s10456-017-9573-6.

[15] S. Telang, B.F. Clem, A.C. Klarer, A.L. Clem, J.O. Trent, R. Bucala, J. Chesney, Small molecule inhibition of 6-phosphofructo-2-kinase suppresses t cell activation, J. Transl. Med. 10 (2012) 1. doi:10.1186/1479-5876-10-95.

[16] S. Boyd, J.L. Brookfield, S.E. Critchlow, I.A. Cumming, N.J. Curtis, J. Debreczeni, S.L. Degorce, C. Donald, N.J. Evans, S. Groombridge, P. Hopcroft, N.P. Jones, J.G. Kettle, S. Lamont, H.J. Lewis, P. MacFaull, S.B. McLoughlin, L.J.M. Rigoreau, J.M. Smith, S. St-Gallay, J.K. Stock, A.P. Turnbull, E.R. Wheatley, J. Winter, J. Wingfield, Structurebased design of potent and selective inhibitors of the metabolic kinase PFKFB3, J. Med. Chem. 58 (2015) 3611-3625. doi:10.1021/acs.jmedchem.5b00352.

[17] W. Zhu, L. Ye, J. Zhang, P. Yu, H. Wang, Z. Ye, J. Tian, PFK15, a Small Molecule Inhibitor of PFKFB3, Induces Cell Cycle Arrest, Apoptosis and Inhibits Invasion in Gastric Cancer, PLoS One. 11 (2016) e0163768. doi:10.1371/journal.pone.0163768.

[18] B.F. Clem, J. O'Neal, G. Tapolsky, A.L. Clem, Y. Imbert-Fernandez, D.A. Kerr, A.C. Klarer, R. Redman, D.M. Miller, J.O. Trent, S. Telang, J. Chesney, Targeting 6Phosphofructo-2-Kinase (PFKFB3) as a Therapeutic Strategy against Cancer, Mol. Cancer Ther. 12 (2013) 1461-1470. doi:10.1158/1535-7163.MCT-13-0097.

[19] D. Bouïs, G.A.P. Hospers, C. Meijer, G. Molema, N.H. Mulder, Endothelium in vitro: A 
review of human vascular endothelial cell lines for blood vessel-related research, Angiogenesis. 4 (2001) 91-102. doi:10.1023/A:1012259529167.

[20] F. Hirschhaeuser, U.G.A. Sattler, W. Mueller-Klieser, Lactate: a metabolic key player in cancer., Cancer Res. 71 (2011) 6921-5. doi:10.1158/0008-5472.CAN-11-1457.

[21] S. Romero-Garcia, M.M.B. Moreno-Altamirano, H. Prado-Garcia, F.J. SánchezGarcía, Lactate contribution to the tumor microenvironment: Mechanisms, effects on immune cells and therapeutic relevance, Front. Immunol. 7 (2016) 1-11. doi:10.3389/fimmu.2016.00052.

[22] F. Végran, R. Boidot, C. Michiels, P. Sonveaux, O. Feron, Lactate influx through the endothelial cell monocarboxylate transporter MCT1 supports an NF-kB/IL-8 pathway that drives tumor angiogenesis, Cancer Res. 71 (2011) 2550-2560. doi:10.1158/00085472.CAN-10-2828.

[23] J. Pérez-Escuredo, V.F. Van Hée, M. Sboarina, J. Falces, V.L. Payen, L. Pellerin, P. Sonveaux, Monocarboxylate transporters in the brain and in cancer, Biochim. Biophys. Acta - Mol. Cell Res. 1863 (2016) 2481-2497. doi:10.1016/j.bbamcr.2016.03.013.

[24] B. Cruys, B.W. Wong, A. Kuchnio, D. Verdegem, A.R. Cantelmo, L.C. Conradi, S. Vandekeere, A. Bouche, I. Cornelissen, S. Vinckier, R.M.H. Merks, E. Dejana, H. Gerhardt, M. Dewerchin, K. Bentley, P. Carmeliet, Glycolytic regulation of cell rearrangement in angiogenesis, Nat. Commun. 7 (2016) 1-15. doi:10.1038/ncomms 12240 .

[25] K. De Bock, M. Georgiadou, P. Carmeliet, Role of endothelial cell metabolism in vessel sprouting, Cell Metab. 18 (2013) 634-647. doi:10.1016/j.cmet.2013.08.001.

[26] M. Nguyen, J. Arkell, C.J. Jackson, Human endothelial gelatinases and angiogenesis, Int. J. Biochem. Cell Biol. 33 (2001) 960-970. doi:10.1016/S1357-2725(01)00007-3.

[27] D. Hanahan, R.A. Weinberg, Hallmarks of cancer: the next generation., Cell. 144 (2011) 646-74. doi:10.1016/j.cell.2011.02.013.

[28] C. Costa, J. Incio, R. Soares, Angiogenesis and chronic inflammation: cause or consequence?, Angiogenesis. 10 (2007) 149-166. doi:10.1007/s10456-007-9074-0.

[29] C.S. Abhinand, R. Raju, S.J. Soumya, P.S. Arya, P.R. Sudhakaran, VEGF-A/VEGFR2 signaling network in endothelial cells relevant to angiogenesis, J. Cell Commun. Signal. 10 (2016) 347-354. doi:10.1007/s12079-016-0352-8.

[30] D. Basagiannis, S. Zografou, C. Murphy, T. Fotsis, L. Morbidelli, M. Ziche, C. Bleck, J. Mercer, S. Christoforidis, VEGF induces signalling and angiogenesis by directing VEGFR2 internalisation via macropinocytosis., J. Cell Sci. (2016) jcs.188219. doi:10.1242/jcs.188219.

[31] M. Shibuya, Vascular Endothelial Growth Factor (VEGF) and Its Receptor (VEGFR) Signaling in Angiogenesis: A Crucial Target for Anti- and Pro-Angiogenic Therapies, Genes and Cancer. 2 (2011) 1097-1105. doi:10.1177/1947601911423031.

[32] R.C.A. Sainson, D.A. Johnston, H.C. Chu, M.T. Holderfield, M.N. Nakatsu, S.P. Crampton, J. Davis, E. Conn, C.C.W. Hughes, TNF primes endothelial cells for angiogenic sprouting by inducing a tip cell phenotype., Blood. 111 (2008) 4997-5007. doi:10.1182/blood-2007-08-108597.

[33] P. Vanderslice, C.L. Munsch, E. Rachal, D. Erichsen, K.M. Sughrue, A.N. Truong, J.N. Wygant, B.W. McIntyre, S.G. Eskin, R.G. Tilton, P.J. Polverini, Angiogenesis induced by tumor necrosis factor-a; is mediated by a4 integrins, Angiogenesis. 2 (1998) 265275. doi:10.1023/A:1009296700991.

[34] J. Karar, A. Maity, PI3K/AKT/mTOR Pathway in Angiogenesis, Front. Mol. Neurosci. 4 (2011) 51. doi:10.3389/fnmol.2011.00051.

[35] R. Zhang, Y. Xu, N. Ekman, Z. Wu, J. Wu, K. Alitalo, W. Min, Etk/Bmx transactivates vascular endothelial growth factor 2 and recruits phosphatidylinositol 3-kinase to mediate the tumor necrosis factor-induced angiogenic pathway., J. Biol. Chem. 278 (2003) 51267-76. doi:10.1074/jbc.M310678200.

[36] G. Ferrari, B. Cook, V. Terushkin, G. Pintucci, P. Mignatti, Transforming Growth Factor-beta 1 (TGF- $\beta 1$ ) induces angiogenesis through Vascular Endothelial Growth Factor (VEGF)-mediated apoptosis, J Cell Physiol. 219 (2009) 449-458. 
doi:10.1002/jcp.21706.

[37] P.A. Guerrero, J.H. McCarty, TGF- $\beta$ Activation and Signaling in Angiogenesis, in: Physiol. Pathol. Angiogenes. - Signal. Mech. Target. Ther., InTech, 2017: p. 7. doi:10.5772/66405.

[38] N. Nishida, H. Yano, T. Nishida, T. Kamura, M. Kojiro, Angiogenesis in cancer, Vasc. Health Risk Manag. 2 (2006) 213-219. doi:10.2147/vhrm.2006.2.3.213.

[39] C. Camaré, M. Pucelle, A. Nègre-Salvayre, R. Salvayre, Angiogenesis in the atherosclerotic plaque, Redox Biol. 12 (2017) 18-34. doi:10.1016/j.redox.2017.01.007.

[40] M. Potente, H. Gerhardt, P. Carmeliet, Basic and therapeutic aspects of angiogenesis, Cell. 146 (2011) 873-887. doi:10.1016/j.cell.2011.08.039.

[41] R.S. Kerbel, Issues regarding improving the impact of antiangiogenic drugs for the treatment of breast cancer, The Breast. 18 (2009) S41-S47. doi:10.1016/S09609776(09)70271-1.

[42] J.M. Clarke, H.I. Hurwitz, Understanding and targeting resistance to anti-angiogenic therapies., J. Gastrointest. Oncol. 4 (2013) 253-63. doi:10.3978/j.issn.20786891.2013.036.

[43] D. Gramatzki, P. Roth, E.J. Rushing, J. Weller, N. Andratschke, S. Hofer, D. Korol, L. Regli, A. Pangalu, M. Pless, J. Oberle, R. Bernays, H. Moch, S. Rohrmann, M. Weller, Bevacizumab may improve quality of life, but not overall survival in glioblastoma: an epidemiological study, Ann. Oncol. 29 (2018) 1431-1436. doi:10.1093/annonc/mdy106.

[44] D. Verdegem, S. Moens, P. Stapor, P. Carmeliet, Endothelial cell metabolism: parallels and divergences with cancer cell metabolism, Cancer Metab. 2 (2014) 19. doi:10.1186/2049-3002-2-19.

[45] R.L. Aft, F.W. Zhang, D. Gius, Evaluation of 2-deoxy-D-glucose as a chemotherapeutic agent: mechanism of cell death., Br. J. Cancer. 87 (2002) 805-12. doi:10.1038/sj.bjc.6600547.

[46] I. Ben Sahra, K. Laurent, S. Giuliano, F. Larbret, G. Ponzio, P. Gounon, Y. Le Marchand-Brustel, S. Giorgetti-Peraldi, M. Cormont, C. Bertolotto, M. Deckert, P. Auberger, J.-F. Tanti, F. Bost, Targeting cancer cell metabolism: the combination of metformin and 2-deoxyglucose induces p53-dependent apoptosis in prostate cancer cells., Cancer Res. 70 (2010) 2465-75. doi:10.1158/0008-5472.CAN-09-2782.

[47] B. Emini Veseli, P. Perrotta, P. Van Wielendaele, A.M. Lambeir, A. Abdali, S. Bellosta, G. Monaco, G. Bultynck, W. Martinet, G.R.Y. De Meyer, Small molecule 3PO inhibits glycolysis but does not bind to 6-phosphofructo-2-kinase/fructose-2,6-bisphosphatase3 (PFKFB3), FEBS Lett. 594 (2020) 3067-3075. doi:10.1002/1873-3468.13878.

[48] P. Chand, G.H. Tapolsky, Pfkfb3 inhibitor and methods of use as an anti-cancer therapeutic, 2013. https://patents.google.com/patent/WO2013148228A1.

[49] G. Baek, Y.F. Tse, Z. Hu, D. Cox, N. Buboltz, P. McCue, C.J. Yeo, M.A. White, R.J. DeBerardinis, E.S. Knudsen, A.K. Witkiewicz, MCT4 Defines a Glycolytic Subtype of Pancreatic Cancer with Poor Prognosis and Unique Metabolic Dependencies, Cell Rep. 9 (2014) 2233-2249. doi:10.1016/J.CELREP.2014.11.025.

[50] R.A. Noble, N. Bell, H. Blair, A. Sikka, H. Thomas, N. Phillips, S. Nakjang, S. Miwa, R. Crossland, V. Rand, D. Televantou, A. Long, H.C. Keun, C.M. Bacon, S. Bomken, S.E. Critchlow, S.R. Wedge, Inhibition of monocarboxyate transporter 1 by AZD3965 as a novel therapeutic approach for diffuse large B-cell lymphoma and burkitt lymphoma, Haematologica. 102 (2017) 1247-1257. doi:10.3324/haematol.2016.163030.

[51] A. Minchenko, I. Leshchinsky, I. Opentanova, N. Sang, V. Srinivas, V. Armstead, J. Caro, Hypoxia-inducible factor-1-mediated expression of the 6-phosphofructo-2kinase/fructose-2,6-bisphosphatase-3 (PFKFB3) gene: Its possible role in the warburg effect, J. Biol. Chem. 277 (2002) 6183-6187. doi:10.1074/jbc.M110978200.

[52] J. Chesney, J. Clark, A.C. Klarer, Y. Imbert-Fernandez, A.N. Lane, S. Telang, J. Chesney, J. Clark, A.C. Klarer, Y. Imbert-Fernandez, A.N. Lane, S. Telang, Fructose2,6-Bisphosphate synthesis by 6-Phosphofructo-2-Kinase/Fructose-2,6Bisphosphatase 4 (PFKFB4) is required for the glycolytic response to hypoxia and 
tumor growth, Oncotarget. 5 (2014) 6670-6686. doi:10.18632/oncotarget.2213.

[53] Z. Tan, N. Xie, S. Banerjee, H. Cui, M. Fu, V.J. Thannickal, G. Liu, The monocarboxylate transporter 4 is required for glycolytic reprogramming and inflammatory response in macrophages, J. Biol. Chem. 290 (2015) 46-55. doi:10.1074/jbc.M114.603589.

[54] J.R. Doherty, C. Yang, K.E.N. Scott, M.D. Cameron, M. Fallahi, W. Li, M.A. Hall, A.L. Amelio, J.K. Mishra, F. Li, M. Tortosa, H.M. Genau, R.J. Rounbehler, Y. Lu, C. V. Dang, K.G. Kumar, A.A. Butler, T.D. Bannister, A.T. Hooper, K. Unsal-Kacmaz, W.R. Roush, J.L. Cleveland, Blocking lactate export by inhibiting the myc target MCT1 disables glycolysis and glutathione synthesis, Cancer Res. 74 (2014) 908-920. doi:10.1158/0008-5472.CAN-13-2034.

[55] A.R. Cantelmo, L.C. Conradi, A. Brajic, J. Goveia, J. Kalucka, A. Pircher, P. Chaturvedi, J. Hol, B. Thienpont, L.A. Teuwen, S. Schoors, B. Boeckx, J. Vriens, A. Kuchnio, K. Veys, B. Cruys, L. Finotto, L. Treps, T.E. Stav-Noraas, F. Bifari, P. Stapor, I. Decimo, K. Kampen, K. De Bock, G. Haraldsen, L. Schoonjans, T. Rabelink, G. Eelen, B. Ghesquière, J. Rehman, D. Lambrechts, A.B. Malik, M. Dewerchin, P. Carmeliet, Inhibition of the Glycolytic Activator PFKFB3 in Endothelium Induces Tumor Vessel Normalization, Impairs Metastasis, and Improves Chemotherapy, Cancer Cell. 30 (2016) 968-985. doi:10.1016/j.ccell.2016.10.006.

[56] Y. Cao, X. Zhang, L. Wang, Q. Yang, Q. Ma, J. Xu, J. Wang, L. Kovacs, R.J. Ayon, Z. Liu, M. Zhang, Y. Zhou, X. Zeng, Y. Xu, Y. Wang, D.J. Fulton, N.L. Weintraub, R. Lucas, Z. Dong, J.X.J. Yuan, J.C. Sullivan, L. Meadows, S.A. Barman, C. Wu, J. Quan, M. Hong, Y. Su, Y. Huo, PFKFB3-mediated endothelial glycolysis promotes pulmonary hypertension, Proc. Natl. Acad. Sci. U. S. A. 116 (2019) 13394-13403. doi:10.1073/pnas.1821401116.

[57] R. Zhang, R. Li, Y. Liu, L. Li, Y. Tang, The Glycolytic Enzyme PFKFB3 Controls TNFa-Induced Endothelial Proinflammatory Responses, Inflammation. 42 (2019) 146-155. doi:https://doi.org/10.1007/s10753-018-0880-x.

[58] U. Harjes, K. Bensaad, A.L. Harris, Endothelial cell metabolism and implications for cancer therapy, Br. J. Cancer. 107 (2012) 1207-1212. doi:10.1038/bjc.2012.398.

[59] T. Yamamoto, N. Takano, K. Ishiwata, M. Ohmura, Y. Nagahata, T. Matsuura, A. Kamata, K. Sakamoto, T. Nakanishi, A. Kubo, T. Hishiki, M. Suematsu, Reduced methylation of PFKFB3 in cancer cells shunts glucose towards the pentose phosphate pathway, Nat. Commun. 5 (2014) 3480. doi:10.1038/ncomms4480.

[60] M. Seo, Y.-H. Lee, PFKFB3 regulates oxidative stress homeostasis via its Sglutathionylation in cancer., J. Mol. Biol. 426 (2014) 830-42. doi:10.1016/j.jmb.2013.11.021.

[61] S. Hao, NADPH promotes the rapid growth of the tumor, Infect. Int. 6 (2018) 57-60. doi:10.1515/ii-2017-0164.

[62] P. Vizán, S. Sánchez-Tena, G. Alcarraz-Vizán, M. Soler, R. Messeguer, M.D. Pujol, W.N.P. Lee, M. Cascante, Characterization of the metabolic changes underlying growth factor angiogenic activation: Identification of new potential therapeutic targets, Carcinogenesis. 30 (2009) 946-952. doi:10.1093/carcin/bgp083.

[63] C.C. Alano, P. Garnier, W. Ying, Y. Higashi, T.M. Kauppinen, R.A. Swanson, NAD+ depletion is necessary and sufficient for poly(ADP-ribose) polymerase-1-mediated neuronal death, J. Neurosci. 30 (2010) 2967-2978. doi:10.1523/JNEUROSCI.555209.2010.

[64] W. Ying, C. Alano, P. Garnier, R. Swanson, NAD+ as a metabolic link between DNA damage and cell death, J. Neurosci. Res. 79 (2004) 216-223. doi:https://doi.org/10.1002/jnr.20289.

[65] N. Preyat, M. Rossi, J. Kers, L. Chen, J. Bertin, P.J. Gough, A. Le Moine, A. Rongvaux, F. Van Gool, O. Leo, Intracellular nicotinamide adenine dinucleotide promotes TNF-induced necroptosis in a sirtuin-dependent manner, Cell Death Differ. 23 (2016) 29-40. doi:10.1038/cdd.2015.60.

[66] M. Mericskay, Nicotinamide adenine dinucleotide homeostasis and signalling in heart 
disease: Pathophysiological implications and therapeutic potential, Arch. Cardiovasc. Dis. 109 (2016) 207-215. doi:10.1016/j.acvd.2015.10.004.

[67] K. Yaku, K. Okabe, K. Hikosaka, T. Nakagawa, NAD metabolism in cancer therapeutics, Front. Oncol. 8 (2018) 1-9. doi:10.3389/fonc.2018.00622.

[68] Y.S. Elhassan, A.A. Philp, G.G. Lavery, Targeting NAD+ in metabolic disease: New insights into an old molecule, J. Endocr. Soc. 1 (2017) 816-835. doi:10.1210/js.201700092.

[69] C. Granchi, D. Fancelli, F. Minutolo, An update on therapeutic opportunities offered by cancer glycolytic metabolism, Bioorg. Med. Chem. Lett. 24 (2014) 4915-4925. doi:10.1016/J.BMCL.2014.09.041.

[70] K. Poels, J.G. Schnitzler, F. Waissi, J.H.M. Levels, E.S.G. Stroes, M.J.A.P. Daemen, E. Lutgens, A.M. Pennekamp, D.P.V. De Kleijn, T.T.P. Seijkens, J. Kroon, Inhibition of PFKFB3 Hampers the Progression of Atherosclerosis and Promotes Plaque Stability, Front. Cell Dev. Biol. 8 (2020) 1-11. doi:10.3389/fcell.2020.581641.

[71] C. De Dominicis, P. Perrotta, S. Dall'Angelo, L. Wyffels, S. Staelens, G.R.Y. De Meyer, M. Zanda, ZCDD083: A PFKFB3-Targeted PET Tracer for Atherosclerotic Plaque Imaging, ACS Med. Chem. Lett. 11 (2020) 933-939. doi:10.1021/acsmedchemlett.9b00677.

[72] R. Quarck, M. Wynants, E. Verbeken, B. Meyns, M. Delcroix, Contribution of inflammation and impaired angiogenesis to the pathobiology of chronic thromboembolic pulmonary hypertension, Eur. Respir. J. 46 (2015) 431-443. doi:10.1183/09031936.00009914.

[73] M.R. De Vries, P.H.A. Quax, Plaque angiogenesis and its relation to inflammation and atherosclerotic plaque destabilization, Curr. Opin. Lipidol. 27 (2016) 499-506. doi:10.1097/MOL.0000000000000339.

[74] A. Albini, F. Tosetti, R. Benelli, D.M. Noonan, Tumor inflammatory angiogenesis and its chemoprevention, Cancer Res. 65 (2005) 10637-10641. doi:10.1158/00085472.CAN-05-3473.

[75] H. Jiang, H. Shi, M. Sun, Y. Wang, Q. Meng, P. Guo, Y. Cao, J. Chen, X. Gao, E. Li, J. Liu, PFKFB3-Driven Macrophage Glycolytic Metabolism Is a Crucial Component of Innate Antiviral Defense, J. Immunol. $197 \quad$ (2016) 2880-2890. doi:10.4049/jimmunol.1600474.

[76] L. Kovacs, Y. Cao, W. Han, L. Meadows, A. Kovacs-Kasa, D. Kondrikov, A.D. Verin, S.A. Barman, Z. Dong, Y. Huo, Y. Su, PFKFB3 in smooth muscle promotes vascular remodeling in pulmonary arterial hypertension, Am. J. Respir. Crit. Care Med. 200 (2019) 617-627. doi:10.1164/rccm.201812-22900C.

[77] Z. Yang, H. Fujii, S. V. Mohan, J.J. Goronzy, C.M. Weyand, Phosphofructokinase deficiency impairs atp generation, autophagy, and redox balance in rheumatoid arthritis T cells, J. Exp. Med. 210 (2013) 2119-2134. doi:10.1084/jem.20130252.

[78] S. Mondal, D. Roy, S. Sarkar Bhattacharya, L. Jin, D. Jung, S. Zhang, E. Kalogera, J. Staub, Y. Wang, W. Xuyang, A. Khurana, J. Chien, S. Telang, J. Chesney, G. Tapolsky, D. Petras, V. Shridhar, Therapeutic targeting of PFKFB3 with a novel glycolytic inhibitor PFK158 promotes lipophagy and chemosensitivity in gynecologic cancers, Int. J. Cancer. 144 (2019) 178-189. doi:10.1002/ijc.31868.

[79] F. Bernini, S. Bellosta, A. Corsini, F.M. Maggi, R. Fumagalli, A.L. Catapano, Cholesterol stimulation of HDL binding to human endothelial cells EAhy 926 and skin fibroblasts: evidence for a mechanism independent of cellular metabolism, Biochim. Biophys. Acta - Lipids Lipid Metab. 1083 (1991) 94-100. doi:10.1016/00052760(91)90129-6.

[80] I. Giunzioni, A. Bonomo, E. Bishop, S. Castiglioni, A. Corsini, S. Bellosta, Cigarette smoke condensate affects monocyte interaction with endothelium, Atherosclerosis. 234 (2014) 383-390. doi:10.1016/j.atherosclerosis.2014.03.029.

[81] S. Bellosta, D. Via, M. Canavesi, P. Pfister, R. Fumagalli, R. Paoletti, F. Bernini, HMGCoA Reductase Inhibitors Reduce MMP-9 Secretion by Macrophages, Arterioscler. Thromb. Vasc. Biol. 18 (1998) 1671-1678. doi:10.1161/01.ATV.18.11.1671. 
[82] F. Tetzlaff, A. Fischer, Human Endothelial Cell Spheroid-based Sprouting Angiogenesis Assay in Collagen, Elife. 8 (2018). doi:10.21769/BioProtoc.2995.

\section{Figure legends}

a

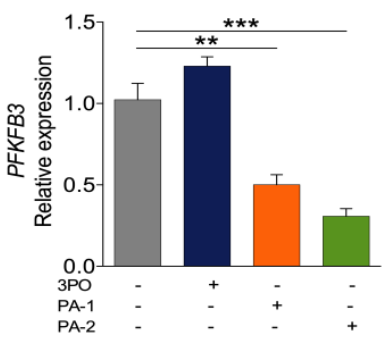

c
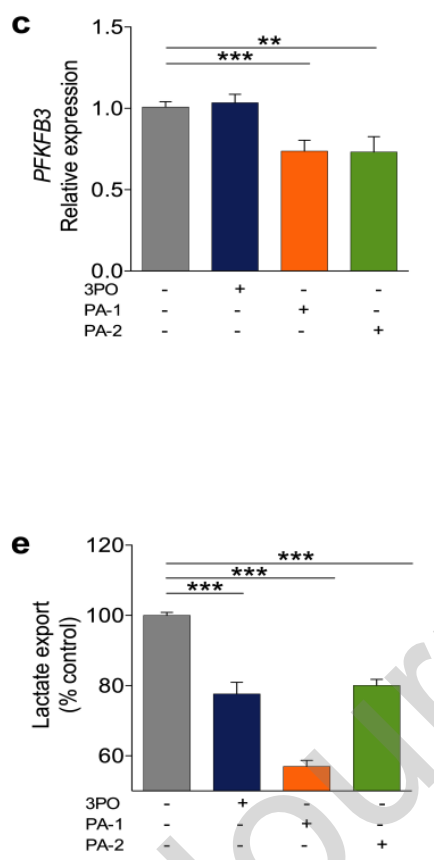

b

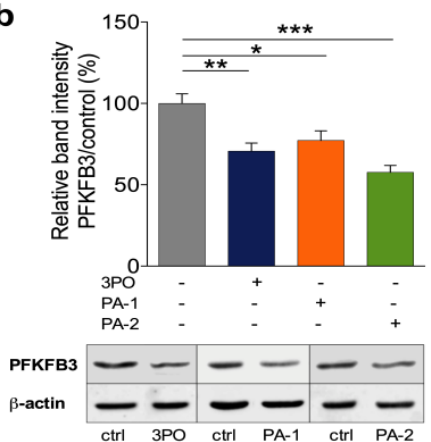

d
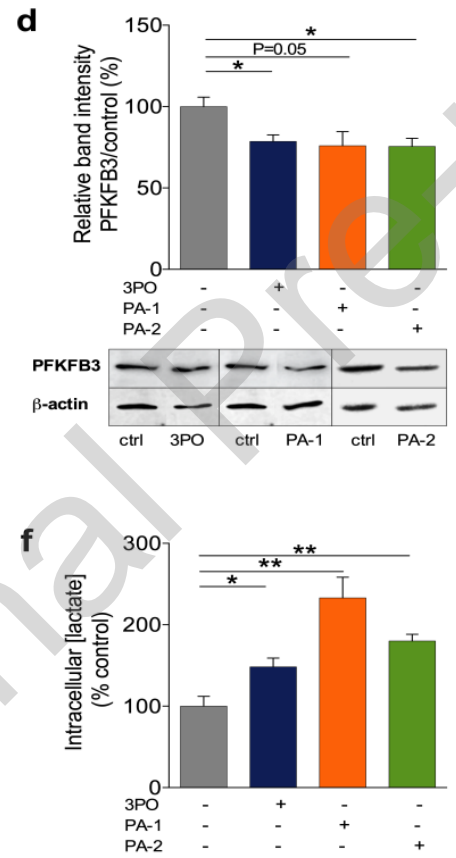

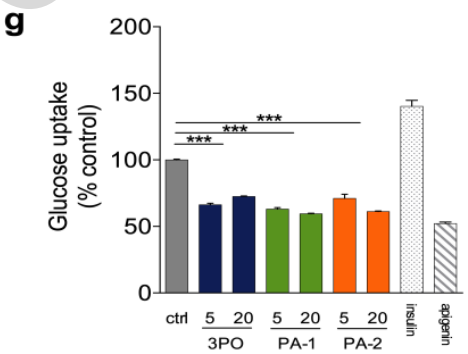

Fig. 1 PA compounds reduce PFKFB3 expression and glycolysis in endothelial cells. (a, b) Treatment with PA-1 and PA-2 reduced the mRNA and protein expression of PFKFB3 in EHECs and in (c, d) HUVECs. (e) Partial reduction of glycolysis (measured by extracellular lactate production) by 3PO, PA-1 and PA-2 in EHECs. (f) Simultaneously, intracellular lactate 
concentration was elevated ( $n=3)$. (g) Glucose uptake using 2-NBDG dye was inhibited after PA treatment. Treatment EHEC: $20 \mu \mathrm{M}$ 3PO, PA-1 and PA-2. Treatment HUVEC: $20 \mu \mathrm{M}$ 3PO and PA-2, 5 $\mu \mathrm{M}$ PA-1. All experiments were performed at least three times. Data are presented as mean \pm SEM, ${ }^{*} \mathrm{P}<0.05,{ }^{* *} \mathrm{P}<0.01,{ }^{* * *} \mathrm{P}<0.001$.

a

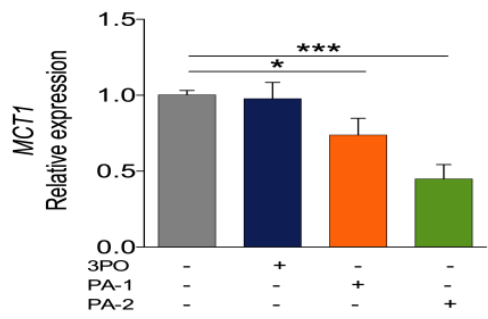

c

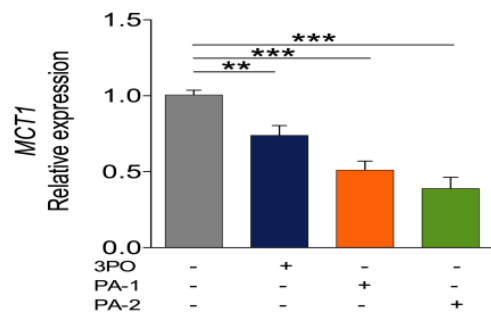

$\mathbf{e}$

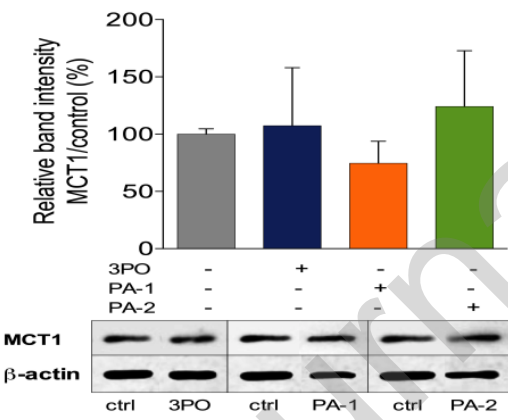

b

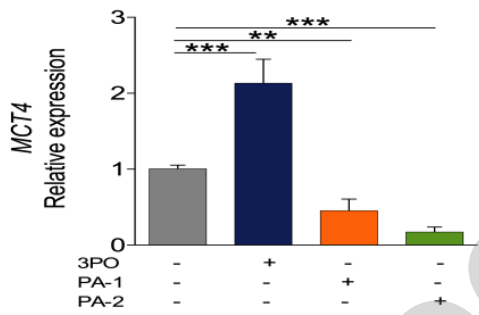

d

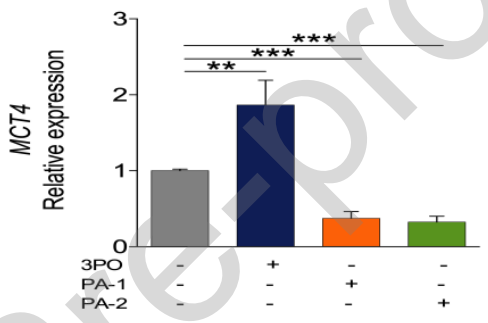

$\mathbf{f}$

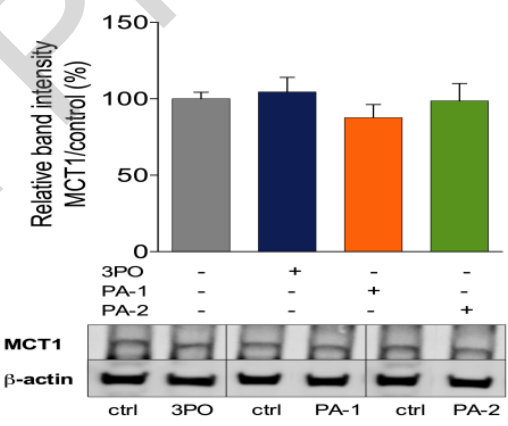

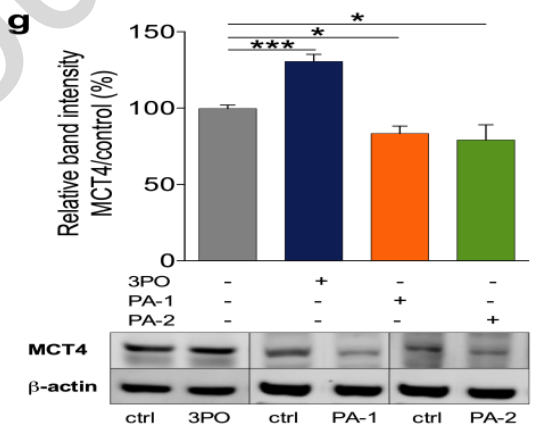

Fig. 2 Endothelial PFKFB3 inhibition reduce MCT1 and MCT4 expression. (a) RT-PCR results show reduced expression of MCT1 and (b) MCT4 in EHECs as well as in (c, d) HUVECs by PA-1 and PA-2. Western blot analysis of MCT1 showing no change of MCT1 protein levels in (e) EHECs and (f) HUVECs in presence of PA compounds. (g) MCT4 protein levels was reduced after PA-1 and PA-2 treatment. Treatment EHEC: $20 \mu \mathrm{M} 3 \mathrm{PO}$, 
PA-1 and PA-2. Treatment HUVEC: $20 \mu \mathrm{M}$ 3PO and PA-2, 5 $\mu \mathrm{M}$ PA-1. All experiments were performed at least three times. Data are presented as mean $\pm \mathrm{SEM},{ }^{*} \mathrm{P}<0.05,{ }^{* *} \mathrm{P}<0.01$, *** $\mathrm{P}<0.001$.

a

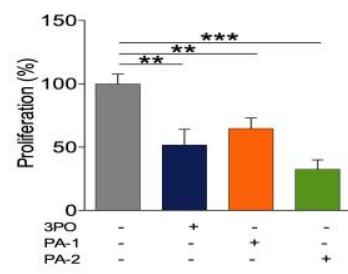

c

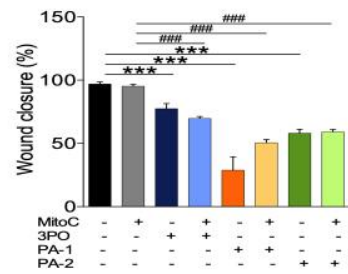

d
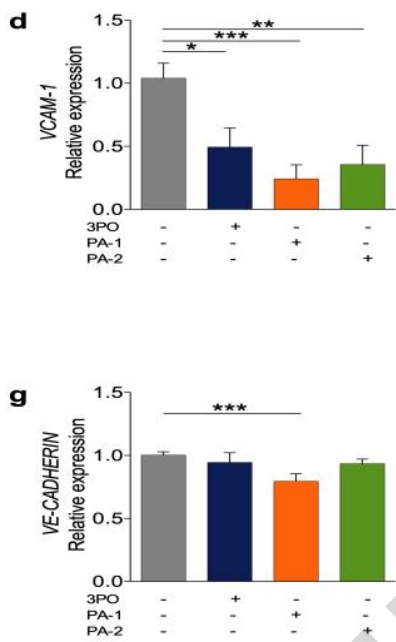

b
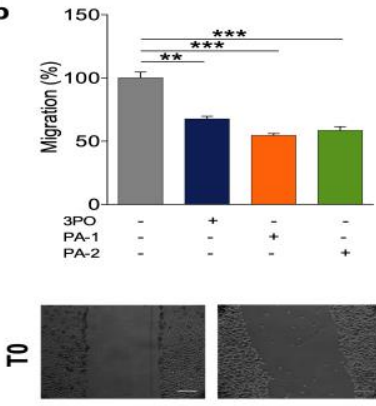

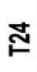

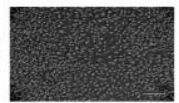

ctrl + MitoC $3 \mathrm{PO}+$ MitoC
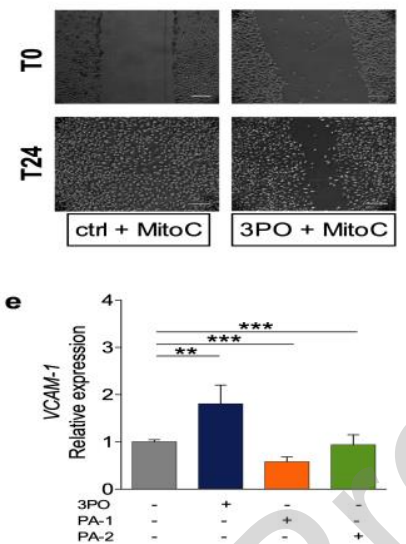

$C$
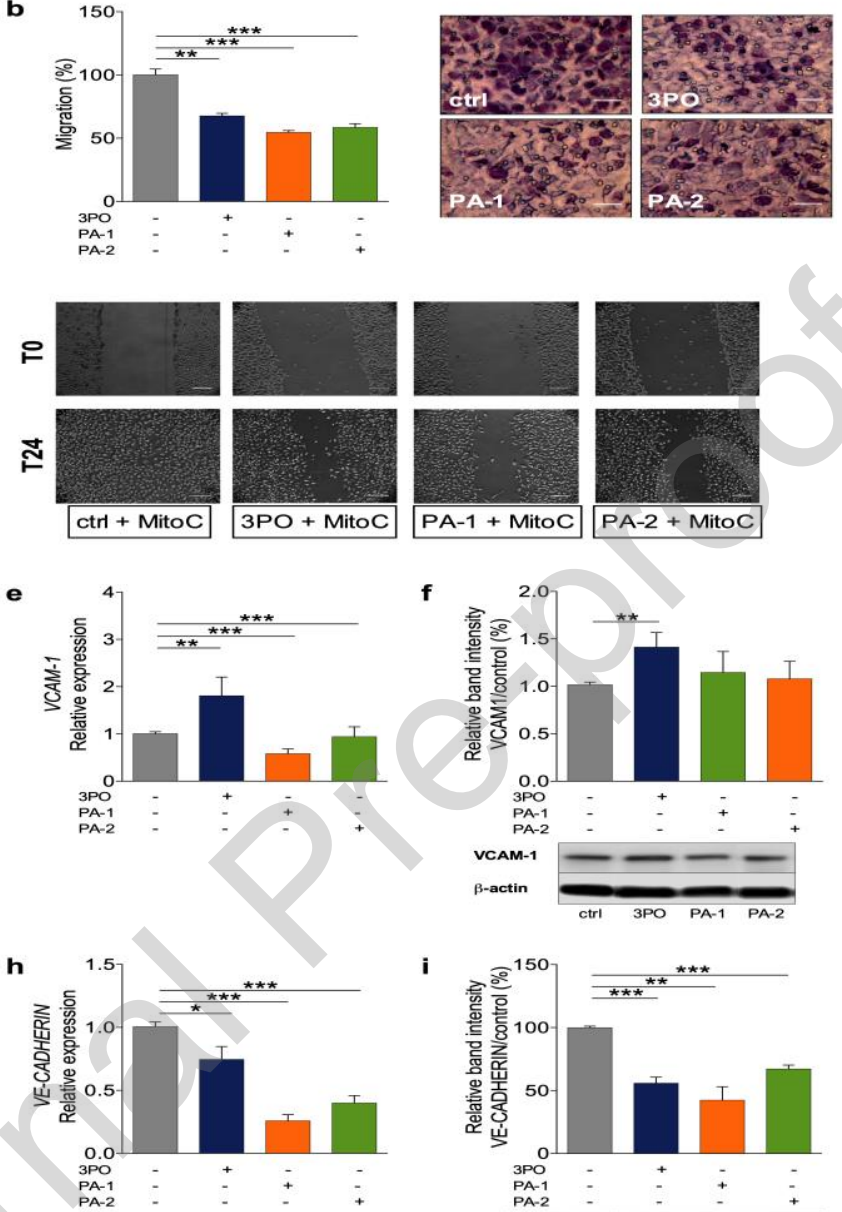

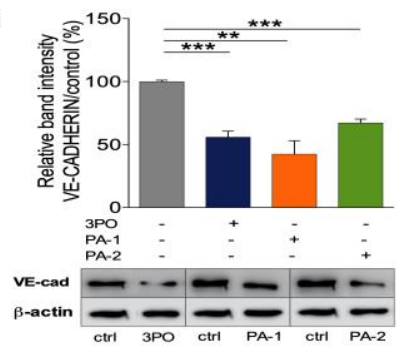

Fig. 3 PFKFB3 inhibition by PA-1 and PA-2 impairs endothelial cell proliferation and migration. (a) PFKFB3 inhibition with PA-1 and PA-2 reduced EC proliferation. (b) Modified Boyden chamber migration assay showing that PFKFB3 inhibition by the PA compounds reduced endothelial migration. Representative phase-contrast images of migrated ECs upon eosin \& tiazine staining (scale bar: $20 \mu \mathrm{m}$ ). (c) Quantification of EC migration in scratch wound assay showing reduced migration upon treatment with PA-1 and PA-2 in absence and presence of MitoC. Representative images of MitoC-treated EC migration in scratch wound assays showing reduced migration upon PFKFB3 inhibition by PA-1 and PA-2 (scale bar: $200 \mu \mathrm{m})$. PA-1 and PA-2 reduced mRNA expression of migration-related gene: VCAM-1 in (d) EHECs and (e) HUVECs. (f) At a protein level, no change was observed in VCAM-1 
levels in HUVECs. Gene expression of VE-cadherin treated with PA compounds in (g) EHECs and (h) HUVECs. (i) Western blot analysis showed reduction of VE-cadherin in

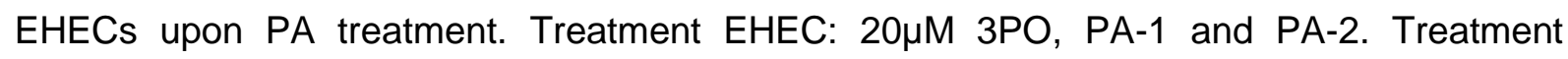
HUVEC: $20 \mu \mathrm{M} 3 \mathrm{PO}$ and PA-2, 5 $\mu \mathrm{M}$ PA-1. All experiments were performed at least three times. Data are represented as mean $\pm \mathrm{SEM},{ }^{*} \mathrm{P}<0.05,{ }^{* *} \mathrm{P}<0.01,{ }^{* *} \mathrm{P}<0.001, \# \# \# \mathrm{P}<$ 0.001

a

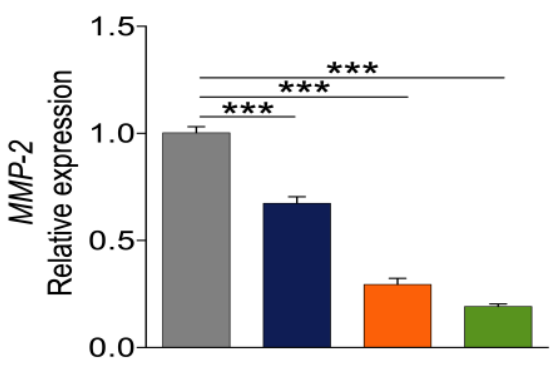

C

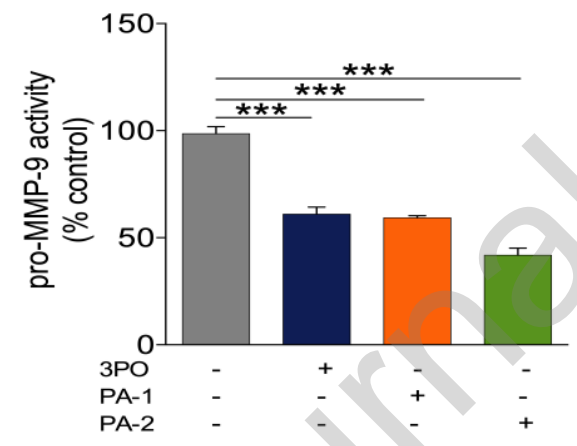

b

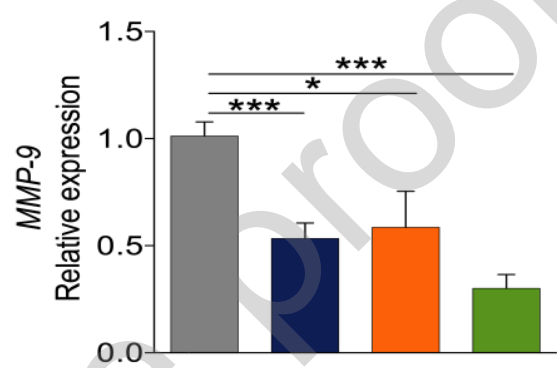

d

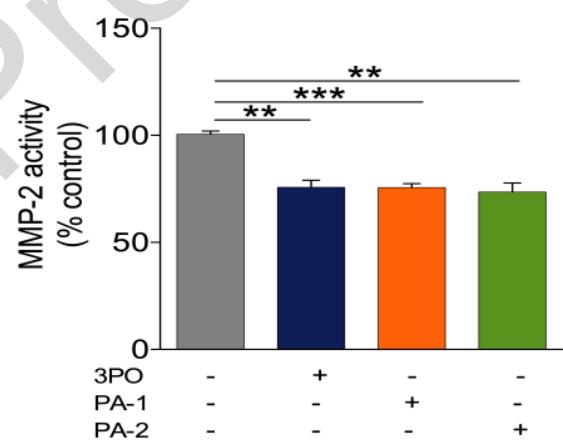

e
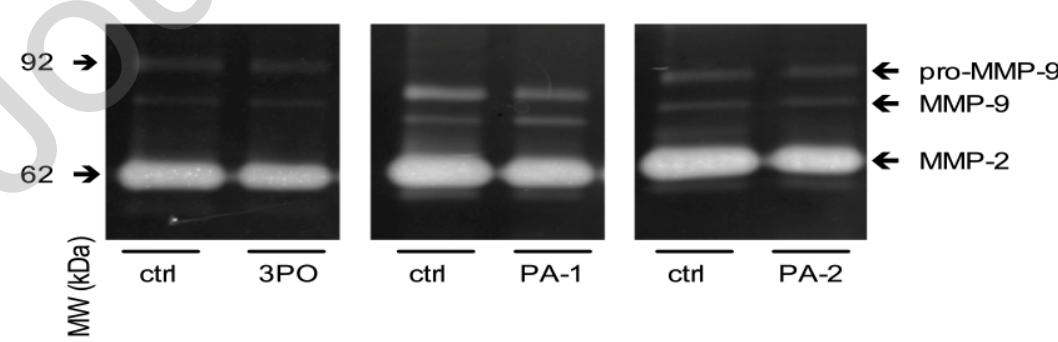

Fig. 4 PFKFB3 inhibition with PA-1 and PA-2 reduce gelatinolytic expression and activity in endothelial cells. (a, b) qRT-PCR results showing reduction of MMP-2 and MMP-9 mRNA levels in EHECs treated with PA-1 and PA-2. (c, d) Gelatin zymography analysis of conditioned medium from EHECs showing reduction of MMP-2 and proMMP-9 activity upon treatment with PA compounds. (e) Representative zymograms of the inhibitory effect of PA compounds on gelatinolytic activity. Treatment EHEC: $20 \mu \mathrm{M} 3 \mathrm{PO}, \mathrm{PA}-1$ and PA-2. All 
experiments were performed at least three times. Data are presented as mean \pm SEM, * $P<$ $0.05,{ }^{* *} \mathrm{P}<0.01,{ }^{* *} \mathrm{P}<0.001$.

a
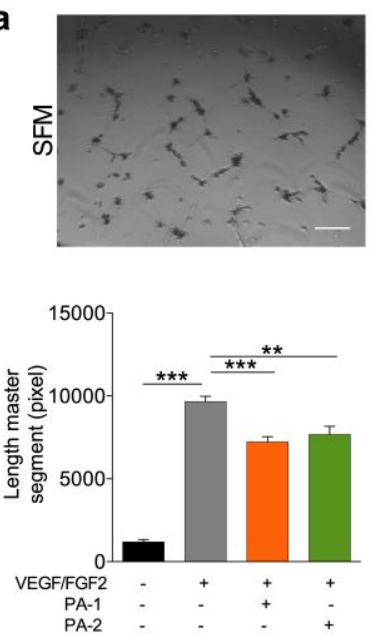

b

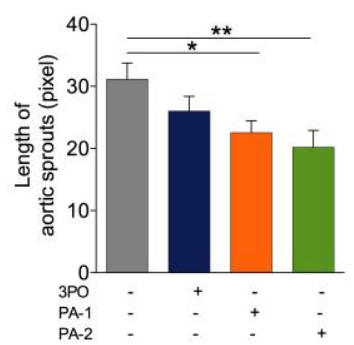

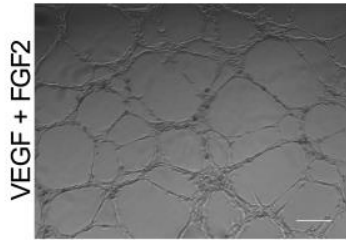
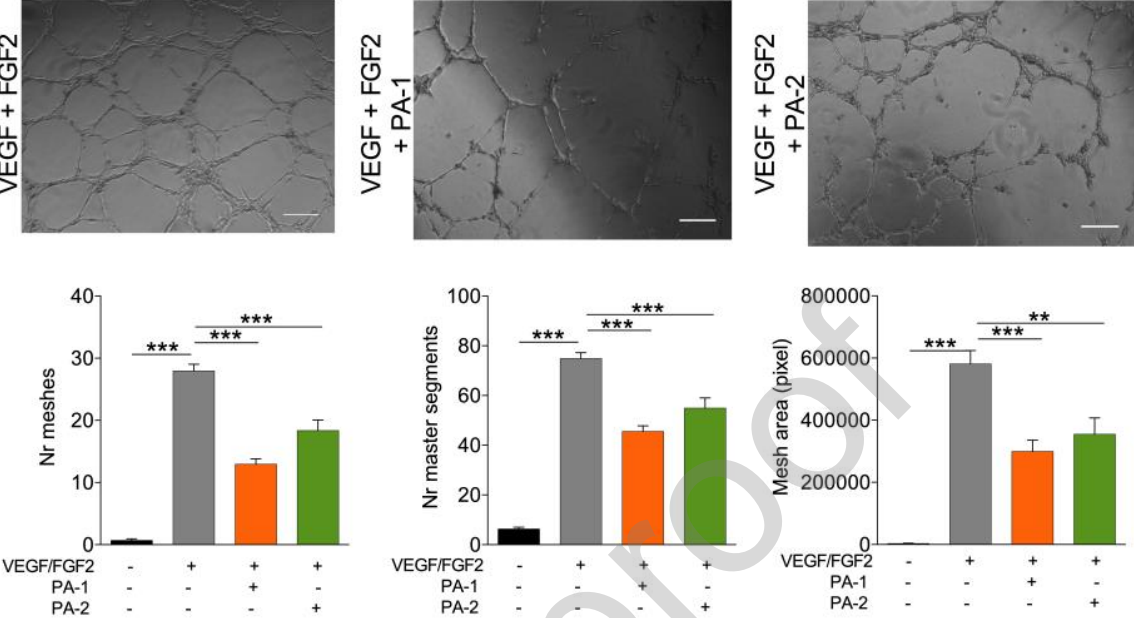

Fig. 5 PFKFB3 inhibition with PA compounds suppress capillary-like structure formation and vessel sprouting in vitro. (a) Morphometric analysis revealing reduced effect of PA-1 and PA2 on the total length of master segments, number of master segments, number of meshes, and mesh area in HUVECs. Representative images showing suppressing effects of PA-1 and PA-2 on in vitro sprouting by HUVECs (scale bar: $200 \mu \mathrm{m}$ ), SFM = serum free medium, i.e. negative control. (b) Morphometric quantification show that PA compounds reduced total

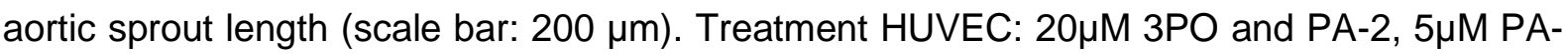
1. All experiments were performed at least three times. Data are presented as mean \pm SEM, ${ }^{*} \mathrm{P}<0.05,{ }^{* *} \mathrm{P}<0.01,{ }^{* * *} \mathrm{P}<0.001$ 
a

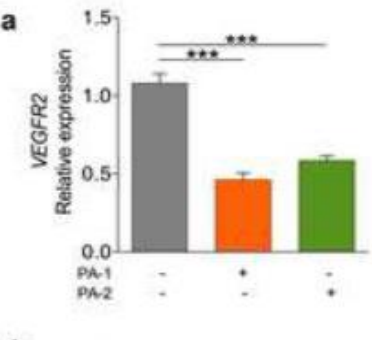

d
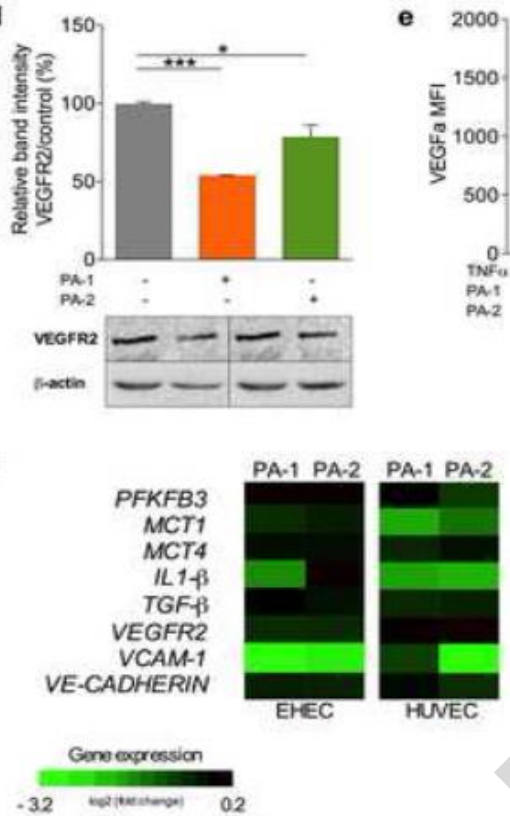

b
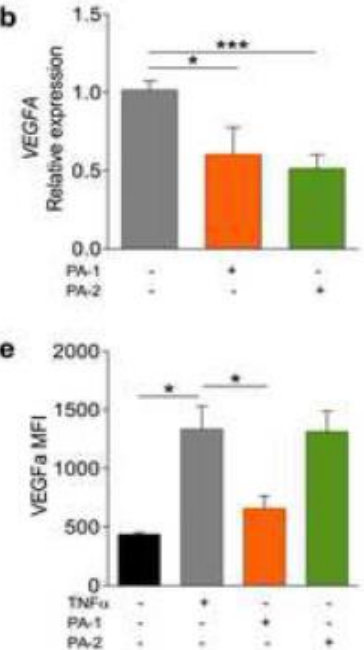

c
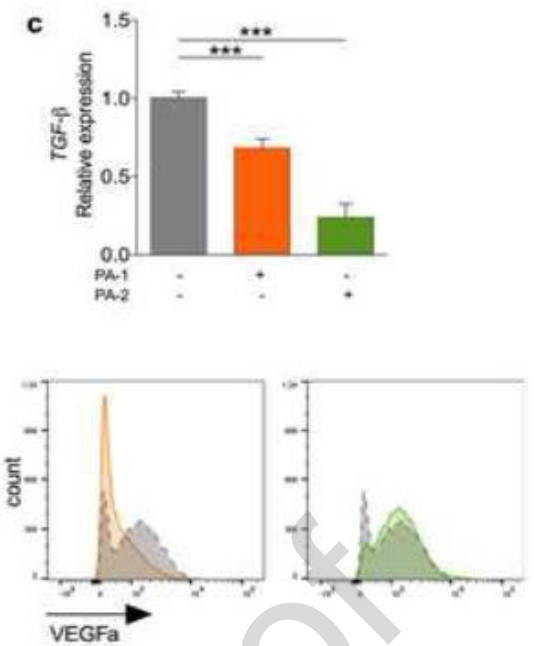

Fig. 6 Endothelial PFKFB3 inhibition with PA compounds act on the VEGFA/VEGFR2 axis in basal and inflammatory conditions. qRT-PCR analysis revealing downregulation of angiogenic genes (a) VEGFR2, (b) VEGFA and (c) TGF- $\beta$ expression in EHECs treated with PA-1 and PA-2. (d) Relative protein expression of VEGFR2 in EHECs showing reduction upon treatment with PA-1 and PA-2. (e) By flow cytometry, a marked reduction of intracellular VEGFA production was observed in HUVECs treated with PA-1. Representative histograms show reduced VEGFA-positive ECs by PA-1 (orange) as compared to control (grey). No change was observed in HUVECs treated with PA-2 (green). (f) Heatmap indicating fold changes in expression levels of genes related to glycolysis, migration, and angiogenesis upon treatment with PA-1 and PA-1 together with TNF- $\alpha$ in EHECs (left) and HUVEC (right). Green color represents downregulation of mRNA levels. Treatment EHEC:

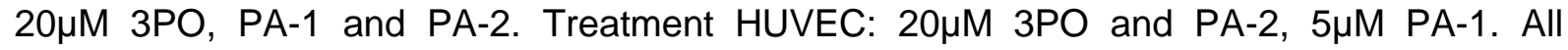
experiments were performed at least three times. Data are represented as mean \pm SEM, * $P$ $<0.05,{ }^{* *} \mathrm{P}<0.01,{ }^{* * *} \mathrm{P}<0.001$ 
Fig. S1 (a) Dose-range effects of PA-1 and PA-2 on endothelial wound closure. A concentration of $20 \mu \mathrm{M}$ PA-1 and PA-2 impaired wound healing function of EHECs. (b) Chemical structures of PFKFB3 inhibitors: PA-1 and PA-2. (c) Dose-range effects of 3PO, PA-1 and PA-2 on endothelial cell viability using LDH cytotoxicity assay. Cytotoxicity was measured on the supernatant of compound-treated EHECs. (d) NAD+ concentration and NAD+/NADH ratio were unchanged upon treatment with PA compounds. Treatment EHEC: $20 \mu \mathrm{M}$ 3PO, PA-1 and PA-2. Data are presented as mean \pm SEM. All experiments were performed at least three times. ${ }^{*} \mathrm{P}<0.05,{ }^{* * *} \mathrm{P}<0.001$

Fig. S2 PFKFB3 inhibition with PA compounds suppress formation of in vitro capillary-like structures by endothelial cells. (a) Quantification of morphometric analysis of in vitro angiogenesis assay showing reduction of several parameters upon treatment with PA-1 and PA-2 in EHECs. (b) Representative images showing suppressing effects of PA-1 and PA-2 on in vitro sprouting by EHECs (scale bar: $200 \mu \mathrm{m}$ ). Treatment EHEC: $20 \mu \mathrm{M}$ PA-1 and PA-2. All experiments were performed at least three times. Data are presented as mean $\pm \mathrm{SEM}$, *** $P<0.001$ 